\title{
Plasma-cascade instability
}

\author{
Vladimir N. Litvinenko® ${ }^{1,2, *}$ Yichao Jing, ${ }^{2,1}$ Dmitry Kayran, ${ }^{2,1}$ Patrick Inacker, ${ }^{2}$ \\ Jun Ma $\odot,{ }^{2}$ Toby Miller, ${ }^{2}$ Irina Petrushina $\odot,{ }^{1}$ Igor Pinayev $\odot,{ }^{2}$ Kai Shih, ${ }^{1}$ \\ Gang Wang, ${ }^{2,1}$ and Yuan $\mathrm{H}$. Wu ${ }^{1}$ \\ ${ }^{1}$ Department of Physics and Astronomy, Stony Brook University, Stony Brook 11794, New York, USA \\ ${ }^{2}$ Collider-Accelerator Department, Brookhaven National Laboratory, Upton 11973, New York, USA
}

(Received 29 October 2020; accepted 8 January 2021; published 27 January 2021)

\begin{abstract}
In this paper we describe a new microbunching instability occurring in charged particle beams propagating along a straight trajectory. The nature of these exponentially growing plasma oscillations gave the reason for its name: plasma-cascade instability. Such instability can strongly amplify longitudinal microbunching originating from the beam's shot noise, even to the point of saturation. Resulting random density and energy microstructures can drastically reduce beam quality. Conversely, such instability can drive novel high-power sources of broadband radiation or can be used as a broadband amplifier. We discovered this phenomenon in a search for such amplifier in the coherent electron cooling scheme [Phys. Rev. Lett. 102, 114801 (2009)] without separation of electron and hadron beams. In this paper we present a brief analytical theory of this new phenomenon, detailed numerical studies, the results of experimental demonstration as well as control of the longitudinal plasma-cascade instability.
\end{abstract}

DOI: 10.1103/PhysRevAccelBeams.24.014402

\section{INTRODUCTION}

High brightness intense charged particle beams play a critical role in the exploration of modern science frontiers [1]. Such beams are central elements of high luminosity hadron colliders [2-5] as well as for x-ray femtosecond free-electron lasers (FEL) [6-20]. In the future, such beams could be necessary for cooling hadron beams in high-luminosity colliders [21-23], for driving x-ray FEL oscillators [24-27] and plasmawakefield accelerators with $\mathrm{TV} / \mathrm{m}$ accelerating gradients [28-36].

Preservation of the beam quality during generation, acceleration, transportation and compression is important for attaining the desirable properties of the beam. Dynamics of high intensity beams are driven by both external factors-such as focusing and accelerating fields - and self-induced (collective) effects—such as space charge [37-58], wakefields from the surrounding environment and radiation of the beam [59-65]. While external factors are designed to preserve beam quality, the collective effects can produce an instability [59] severely

\footnotetext{
*Corresponding author.

vladimir.litvinenko@stonybrook.edu

Published by the American Physical Society under the terms of the Creative Commons Attribution 4.0 International license. Further distribution of this work must maintain attribution to the author(s) and the published article's title, journal citation, and DOI.
}

degrading beam emittance(s), ${ }^{1}$ momentum spread and creating filamentation of the beam. On the other hand, such instabilities can be deliberately built in to attain specific results. The most known application is the FEL instability used for generating coherent radiation from $\mathrm{THz}$ to X-rays [66-70]. Less known applications are coherent electron cooling $(\mathrm{CeC})$ of hadron beams [7175] and generation of broadband high power radiation by microbunched beams [76-78].

The plasma-cascade microbunching instability (PCI) occurs in a beam propagating along a straight line. It is driven by variation of the beam density and corresponding modulation of the beam's plasma frequency [75]. The PCI differs from the conventional microbunching instability (occurring in beams traveling along a curved trajectory ${ }^{2}$ ); which is well known and is studied in depth both theoretically and experimentally [79-97]. Space-chargedriven parametric transverse instabilities are also well known (as discussed in [98] and references therein). But none of them include the PCI-a microbunching longitudinal instability driven by modulations of the transverse beam size or by a bunch compression.

While we theoretically studied many aspects of the PCI including the 3D effects $[75,99]$, this paper is focused on

\footnotetext{
${ }^{1}$ Beam emittance is the phase space area (for $1 \mathrm{D}$ case) or the phase space hypervolume (for 2D and 3D cases) occupied by the beam's particles.

${ }^{2}$ The beam traveling through a magnetic chicane or an arc of an accelerator exemplifies such systems.
} 
the most important and the most practical aspects of such instability, e.g., the longitudinal PCI. We start from a qualitative description of the PCI, which will be followed by a brief theory, 3D simulations and experimental observations of this phenomenon.

\section{QUALITATIVE DESCRIPTION OF THE PHENOMENON IN A COLD PLASMA}

To set the stage, we start with a simple model of plasma oscillations in the rest frame of a homogeneous, infinite and cold beam. Later in the paper we will present more realistic models.

It is known that small density perturbations in a cold homogeneous beam (e.g., non-neutral plasma) will undergo oscillations with plasma frequency $\omega_{p}=c \sqrt{4 \pi n_{o} r_{c}}$, where $n_{o}$ is the particle's density in the comoving beam frame, $c$ is the speed of light, and $r_{c}=e^{2} / m c^{2}$ is the particle's classical radius [100-102], where $e$ is the particle's charge and $m$ is its rest mass. ${ }^{3}$ An equation for the oscillation in a cold plasma can be easily derived from the linearized continuity equation

$$
\frac{\partial n}{\partial t}+\operatorname{div}(n \vec{v})=0
$$

For a confined plasma with uniform stationary density $n_{o}=$ const and infinitesimally small perturbations $\tilde{n}(\vec{r}, t), \frac{|\tilde{n}|}{n_{o}} \ll 1$, differentiating Eq. (1) and using the Maxwell equation for the particle's acceleration,

$$
\frac{\partial \vec{v}}{\partial t}=\frac{e \vec{E}}{m} ; \quad \operatorname{div} \vec{E}=4 \pi e \tilde{n} \rightarrow \operatorname{div} \frac{\partial \vec{v}}{\partial t}=\frac{4 \pi e^{2}}{m} \tilde{n},
$$

we get

$\frac{\partial^{2} \tilde{n}}{\partial t^{2}}+4 \pi \frac{e^{2} n_{o}}{m} \tilde{n}=\left(\frac{\partial \vec{v}}{\partial t} \cdot \vec{\nabla} \tilde{n}+\frac{\partial \tilde{n}}{\partial t} \cdot \operatorname{div} \vec{v}\right)=O\left(\left|\frac{\tilde{n}}{n_{o}}\right|^{2}\right)$

i.e., the well-known equation for plasma oscillations:

$$
\ddot{\tilde{n}}+\omega_{p}^{2} \tilde{n}=0,
$$

having the well-known oscillating solution:

$$
\begin{aligned}
& \tilde{n}=\delta n(\vec{r}) \cdot \cos \left[\omega_{p} t+\varphi(\vec{r})\right] ; \\
& \dot{\tilde{n}}=-\omega_{p} \delta n(\vec{r}) \cdot \sin \left[\omega_{p} t+\varphi(\vec{r})\right],
\end{aligned}
$$

with constants of motion $\delta n, \varphi$ determined by initial conditions for an arbitrary infinitesimal $\delta n(\vec{r}), \delta \dot{n}(\vec{r})$. In other words, each quarter of the plasma period energy of

\footnotetext{
${ }^{3}$ In this paper we are using CGS units. While using $e$ and $m$ is typical for describing electron or proton beams, for charged ion beams one should replace them with $\mathrm{Ze}$ and $\mathrm{Am}$.
}

the plasma oscillations transfer energy from the electrostatic (density perturbation) to the kinetic (velocity perturbation) and vice versa.

The situation does change when the density of the beam and the corresponding plasma frequency vary in time. The time-dependent oscillator equation

$$
\ddot{\tilde{n}}+\omega_{p}^{2}(t) \tilde{n}=0
$$

does not have an analytical solution in general, but it can be represented as a linear symplectic transformation [102-106]

$$
\left[\begin{array}{c}
\tilde{n}\left(t_{2}\right) \\
\dot{\tilde{n}}\left(t_{2}\right)
\end{array}\right]=\mathbf{M}\left(t_{1} \mid t_{2}\right)\left[\begin{array}{l}
\tilde{n}\left(t_{1}\right) \\
\dot{\tilde{n}}\left(t_{1}\right)
\end{array}\right],
$$

which can be formally expressed in the form of an ordered matrix exponent

$\mathbf{M}\left(t_{1} \mid t_{2}\right)=\exp _{\text {ordered }}\left[\int_{t_{1}}^{t_{2}} \mathbf{D}(t) d t\right] ; \quad \mathbf{D}(t)=\left[\begin{array}{cc}0 & 1 \\ -\omega_{p}^{2}(t) & 0\end{array}\right]$,

which corresponds to a matrix calculated as in the following sequence:

$$
\begin{aligned}
& \underset{\text { ordered }}{\exp _{t_{1}}}\left[\int_{t_{2}}^{t_{2}} \mathbf{D}(t)\right] d t=\lim _{N \rightarrow \infty} \prod_{\substack{n=1 \\
\text { ordered }}}^{N} M_{n} \equiv M_{N} \ldots M_{2} M_{1} ; \\
& M_{n}=\exp \left[\mathbf{D}\left(t_{n}\right) \Delta t\right] \\
& =\left[\begin{array}{cc}
\cos \omega_{p}\left(t_{n}\right) \Delta t & \frac{\sin \omega_{p}\left(t_{n}\right) \Delta t}{\omega_{p}\left(t_{n}\right)} \\
-\omega_{p}\left(t_{n}\right) \sin \omega_{p}\left(t_{n}\right) \Delta t & \cos \omega_{p}\left(t_{n}\right) \Delta t
\end{array}\right] ; \\
& \Delta t=\frac{t_{2}-t_{1}}{N} ; \quad t_{n} \in\left\{t_{1}+(n-1) \Delta t, t_{1}+n \Delta t\right\} .
\end{aligned}
$$

with

$$
\operatorname{det} \mathbf{M}\left(t_{1} \mid t_{2}\right)=\exp \int_{t_{1}}^{t_{2}} \operatorname{Trace}[\mathbf{D}(t)] d t=1 .
$$

The resulting matrix can correspond to either stable or unstable oscillations. It is determined by its eigenvalues,

$\operatorname{det}[\mathbf{M}-\lambda \mathbf{I}]=0 ; \quad \mathbf{I}=\left[\begin{array}{ll}1 & 0 \\ 0 & 1\end{array}\right] ; \quad \lambda_{1} \lambda_{2}=\operatorname{det} \mathbf{M}=1$,

which in the general case are unknown functions of $\omega_{p}(t)$ in the interval $\left\{t_{1}, t_{2}\right\}$. In a periodic system with $\omega_{p}(t+T)=\omega_{p}(t)$ the evolution is determined by the "cell" transport matrix:

$\mathbf{M}_{c}=\mathbf{M}(0 \mid T)=\left[\begin{array}{ll}m_{11} & m_{12} \\ m_{21} & m_{22}\end{array}\right] ; \quad \mathbf{M}(0 \mid n T)=\mathbf{M}_{c}{ }^{n} ;$

with stability range determined by the trace of the cell matrix [102-106]: 


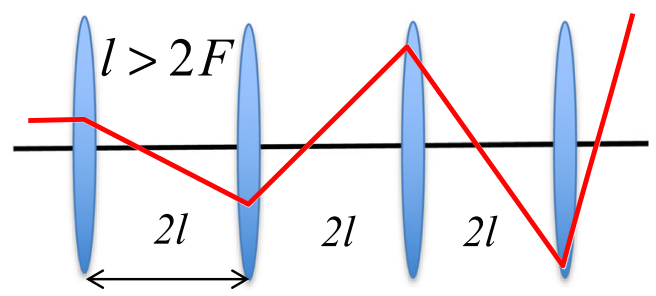

FIG. 1. A sketch of unstable ray trajectory in a system of periodic focusing lenses - an analog of broadband parametric resonance.

$$
-2<\operatorname{Trace}_{c}<2
$$

When $\left|\operatorname{Trace}_{c}\right|>2$, oscillations become unstable ${ }^{4}$ :

$\lambda_{1}=\lambda_{2}{ }^{-1}=\frac{\operatorname{Trace}_{c}}{2}\left(1+\sqrt{1-\frac{4}{\left(\operatorname{Trace}_{c}\right)^{2}}}\right) ; \quad\left|\lambda_{1}\right|>1 ;$

with growing amplitude:

$$
\begin{aligned}
\tilde{n}(n T)= & \tilde{n}(0) \frac{\lambda_{1}{ }^{n}+\lambda_{1}{ }^{-n}}{2} \\
& +\left(m_{12} \dot{\tilde{n}}(0)+\frac{m_{11}-m_{22}}{2} \tilde{n}(0)\right) \frac{\lambda_{1}{ }^{n}-\lambda_{1}{ }^{-n}}{\lambda_{1}-\lambda_{1}{ }^{-1}} ; \\
\dot{\tilde{n}}(n T)= & \dot{\tilde{n}}(0) \frac{\lambda_{1}{ }^{n}+\lambda_{1}{ }^{-n}}{2} \\
& +\left(m_{21} \tilde{n}(0)+\frac{m_{22}-m_{11}}{2} \dot{\tilde{n}}(0)\right) \frac{\lambda_{1}{ }^{n}-\lambda_{1}{ }^{-n}}{\lambda_{1}-\lambda_{1}{ }^{-1}} ;
\end{aligned}
$$

and an asymptotic exponential growth of amplitude of oscillations when $\left|\lambda_{1}^{-n}\right| \ll 1$ :

$$
\begin{aligned}
\tilde{n}(n T) \rightarrow & \tilde{n}(0) \frac{\lambda_{1}{ }^{n}}{2}+\left(m_{12} \dot{\tilde{n}}(0)+\frac{m_{11}-m_{22}}{2} \tilde{n}(0)\right) \\
& \times \frac{\lambda_{1}{ }^{n}}{\lambda_{1}-\lambda_{1}{ }^{-1}} ; \\
\dot{\tilde{n}}(n T) \rightarrow & \dot{\tilde{n}}(0) \frac{\lambda_{1}{ }^{n}}{2}+\left(m_{21} \tilde{n}(0)+\frac{m_{22}-m_{11}}{2} \dot{\tilde{n}}(0)\right) \\
& \times \frac{\lambda_{1}{ }^{n}}{\lambda_{1}-\lambda_{1}{ }^{-1}} ;
\end{aligned}
$$

albeit with alternating sign when $\lambda_{1}<-1$.

It is well known in classical oscillator theory [107] that a modulation of oscillator frequency with a period close to a

\footnotetext{
${ }^{4}$ We understand that stability conditions (13) are well known for readers of this journal. Nevertheless, parametrization of unstable motion needed for this study is less known - this is the reason for repeating some of the otherwise well-known derivations in Eqs. (7)-(12) and adding an Appendix A.
}

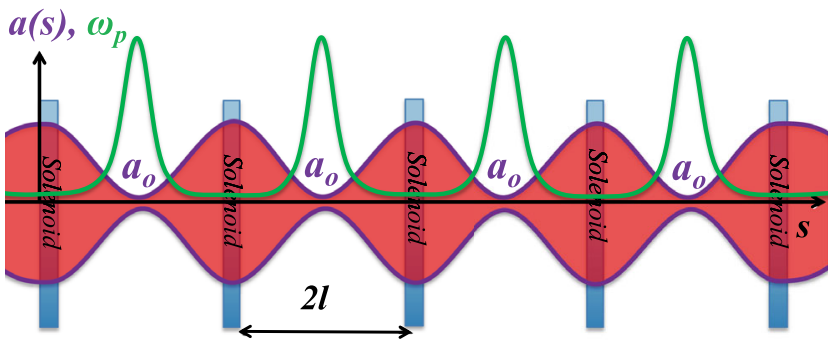

FIG. 2. A sketch of four focusing cells with periodic modulations of beam envelope, $a(s)$, and the plasma frequency, $\omega_{p}$. Beam envelope has waists, $a_{o}$, in the middle of each cell where plasma frequency peaks. Scales are attuned for illustration purposes. The waists of the beam serve as "short focusing elements" for the longitudinal plasma oscillations.

half of the oscillation period would result in an exponential growth of oscillation amplitude, the phenomena known as parametric resonance. Both the width and growth rate of this parametric instability can be enormous when the span of the frequency modulation is large. The extreme case of the $\delta$ function-like modulation is also well known: as illustrated in Fig. 1, a periodic focusing lenses with focal length shorter than a quarter of the separating distances will make rays unstable. The entire half-space $F<l / 2$ is occupied by this parametric resonance with growth rate defined by a negative eigenvalue $\lambda_{1}=-\eta\left(1+\sqrt{1-\eta^{-2}}\right) ; \eta=\frac{l}{F}-1>1$.

These two examples illustrate that modulation of the transverse size, either periodic or aperiodic, could lead to an exponential growth of longitudinal density modulation: e.g., a solution of $s$-dependent Hill's equation [102-106]:

$$
x^{\prime \prime}+K(s) x=0 \quad \text { or } \quad \ddot{\tilde{n}}+\omega_{p}^{2}(t) \tilde{n}=0
$$

can be unstable-here $x^{\prime \prime} \equiv \frac{d^{2} x}{d s^{2}}$ with $s$ being the length of the beam trajectory.

A more realistic and practical case of the density modulation occurs in a charged particle beam propagating through a focusing channel or/and undergoing bunch compression/decompression. A sketch of a beam envelope evolution in a periodic focusing system and the resulting modulation of the plasma frequency are shown in Fig. 2.

Naturally other effects, such as bunch compression (or decompression) as well as bunch acceleration (or deceleration), ${ }^{5}$ would also result in the modulation of the plasma frequency and could result in the amplification of the density and velocity perturbations. In an arbitrary linear accelerator, variation of the density and plasma frequency are generally aperiodic. Therefore, the density and velocity perturbations evolve from the start (cathode) to the exit of

\footnotetext{
${ }^{5}$ Change in the relativistic factor results in corresponding change of the length scale in the comoving frame, e.g., in the effective change of the density. In the lab frame, plasma frequency modulation from the acceleration is the result of the change of its longitudinal mobility.
} 
the accelerator and, in the case of cold infinite homogeneous beam, would be expressed via the transport matrix (8). It is also well known that in plasma with a finite temperature such oscillations will decay because of the Landau damping [108,109] and the perturbations are thermalized $[100,101]$. We are considering these effects later in the paper.

\section{BRIEF THEORETICAL DESCRIPTION OF THE INSTABILITY}

The general theory of plasma cascade instability, including a number of practical cases, is published elsewhere
$[75,99]$. Here we limit our theoretical description to use of the key equations from these papers.

Let us consider first an analytically tractable model for the condition illustrated in Fig. 2. Specifically, let us consider a long round beam propagating along the $z$ axis with constant velocity $v_{o}$ with momentum $p_{o}=\gamma_{o} \beta_{o} m c$ and relativistic parameters $\beta_{o}=\frac{v_{o}}{c} ; \gamma_{o}=\left(1-\beta_{o}^{2}\right)^{-1 / 2}$, and with focusing provided by solenoids, e.g., a system with an axial symmetry. To find a self-consistent solution for transverse motion including the space charge forces, we will consider a transverse Kapchinsky-Vladimirsky (KV) distribution [110]:

$$
\begin{aligned}
f_{\perp}\left(x, x^{\prime}, y, y^{\prime}\right) & =N \cdot \delta\left(\frac{x^{2}+y^{2}}{a(s)^{2}}+\frac{\left[a(s) x^{\prime}-a^{\prime}(s) x\right]^{2}+\left[a(s) y^{\prime}-a^{\prime}(s) y\right]^{2}}{\varepsilon_{K V}{ }^{2}}-1\right) ; \\
a^{\prime}(s) & =\frac{d a(s)}{d s}
\end{aligned}
$$

where $\varepsilon_{k V}$ is the envelope emittance. ${ }^{6}$ The $\mathrm{KV}$ distribution has uniform density inside the beam envelope, $r \leq a(s)$, and zero density outside it. It generates uniform defocusing force and its envelope is described by a second-order nonlinear differential equation [111]:

$$
\begin{gathered}
a^{\prime \prime}+K(s) a-\frac{2}{\beta_{o}{ }^{3} \gamma_{o}{ }^{3}} \frac{I_{o}}{I_{A}} \frac{1}{a}-\frac{\varepsilon_{K V^{2}}}{a^{3}}=0 ; \\
K(s)=\left(\frac{e B_{\mathrm{sol}}(s)}{2 p_{o} c}\right)^{2} \equiv\left(\frac{e B_{\mathrm{sol}}(s)}{2 \beta_{o} \gamma_{o} m c^{2}}\right)^{2},
\end{gathered}
$$

where $K$ is external focusing provided by the solenoid's field $B_{\text {sol }}, I_{o}$ is the beam current and $I_{A}=m c^{3} / e \approx 17 \mathrm{kA}$ is the Alfven current. In the general case, an analytical solution for the beam envelope does not exist and it has to be found using numerical solvers.

The beam density in the laboratory frame,

$$
\rho(z)=\frac{I_{o}}{e_{o} c} \frac{1}{\pi a^{2}(s)}
$$

as well as in the beam comoving (rest) frame,

$$
n_{o}(t)=\frac{I_{o}}{e \beta_{o} \gamma_{o} c} \frac{1}{\pi a^{2}\left(\gamma_{o} \beta_{o} c t\right)},
$$

\footnotetext{
${ }^{6}$ It is worth mentioning that the envelope emittance of the KV distribution is 4 times that of the traditionally defined rms geometric emittance,

$$
\varepsilon_{\mathrm{KV}}=4 \varepsilon_{\mathrm{rms}}
$$

where the emittance $\varepsilon$ describes particles at the edge of the beam and is fourfold larger than the rms beam emittance.
}

can be expressed using the beam current and the solution for the beam envelope. Here we use the Lorentz transformation between the proper time $t$ in the comoving frame and the particle's position in the lab frame, $s$. It can be shown $[75,99]$ that evolution of the high frequency component, $\tilde{f}_{k}(k a \gg 1)$ of a weak perturbation of the longitudinal distribution function $f_{\|}=f_{o}(v)+\tilde{f}(s, v, t)$, $|\tilde{f}|<f_{o}$ ( $v$ is the $z$ component of velocity in the comoving frame) $[75,99,112]$ is described by a linearized set of Vlasov-Poisson equations [113]:

$$
\begin{aligned}
& \frac{\partial}{\partial t} \tilde{f}_{k}+i k v \tilde{f}_{k}+\frac{e E_{s}}{m} \cdot \frac{\partial f_{o}}{\partial v}=0 \\
& i k E_{s}=4 \pi e n_{o}(t) \cdot \int_{-\infty}^{\infty} \tilde{f}_{k}(v, t) d v
\end{aligned}
$$

Assuming a $\kappa-1$ longitudinal velocity distribution $f_{o}(v)=\sigma_{v} / \pi\left(\sigma_{v}{ }^{2}+v^{2}\right)$, with $\sigma_{v}$ being the velocity spread, this set of equations can be reduced to an oscillator equation $[114,115]$ :

$$
\frac{d^{2} \tilde{g}_{k}}{d t^{2}}+\omega_{p}^{2}(t) \tilde{g}_{k}=0 ; \quad \tilde{g}_{k}=\tilde{f}_{k} e^{\left|k \sigma_{v} t\right|}
$$

for density perturbation corrected by the Landau damping $\exp \left(\left|k \sigma_{v} t\right|\right)$ term $[108,109]$

$$
\rho_{k}(t)=\int_{-\infty}^{\infty} \tilde{f}_{k}(v, t) d v=\tilde{g}_{k}(t) \cdot \exp \left(-\left|k \sigma_{v} t\right|\right) .
$$

In other words, the evolution of $\tilde{g}_{k}$ is described by an ordinary second order equation identical to Eq. (6), which is also known as Hill's equation in accelerator literature. As we discussed in a previous section, the solution of Eq. (23) 


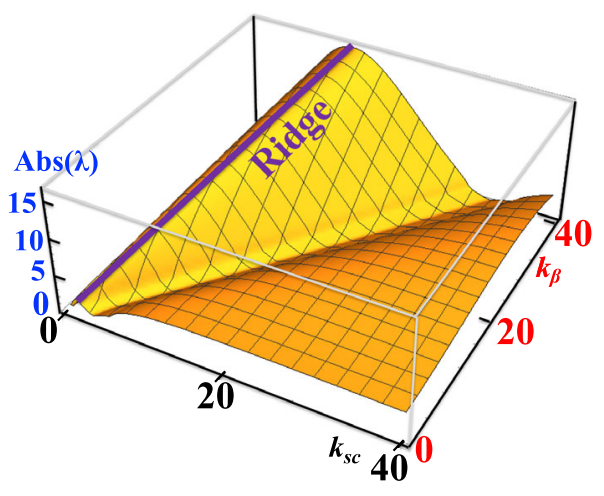

FIG. 3. 3D plot of the absolute value of the growth rate per cell: $\lambda=\max \left(\left|\operatorname{Re} \lambda_{1}\right|,\left|\operatorname{Re} \lambda_{2}\right|\right)$. There is a clearly identifiable ridge along the $k_{\beta} \cong 3 \cdot\left(k_{s c}-1.2\right)$ line, where growth rates peak.

is described by a symplectic ( $\operatorname{det} \mathbf{M}=1$ ) transport matrix. Correcting this solution by Landau damping (24) gives the evolution of the density modulation in such a system.

\section{A. Periodic lattice}

While there is no analytical solution for a general timedependent equation (23), many details can be found for periodic symmetric structures such as that shown in Fig. 2. In this case, equations for the beam envelope and $\tilde{g}_{k}$ can be reduced to a set of dimensionless equations $[75,99]^{7}$

$$
\begin{array}{rlrl}
\frac{d^{2} \hat{a}}{d \hat{s}^{2}}-k_{s c}{ }^{2} \hat{a}^{-1}-k_{\beta}{ }^{2} \hat{a}^{-3} & =0 ; & & \\
\frac{d^{2}}{d \hat{s}^{2}} \tilde{g}_{k}+2 \frac{k_{s c}^{2}}{\hat{a}(\hat{s})^{2}} \cdot \tilde{g}_{k} & =0 ; & \hat{a}=\frac{a}{a_{o}} \geq 1 ; \\
\hat{s} & =\frac{s}{l} ; & \hat{s} \in\{-1,1\},
\end{array}
$$

where $a_{o}$ is the waist of the beam envelope in the cell center $(\hat{s}=0)$, and the evolution of both the envelope and the density perturbations is determined by two dimensionless parameters:

$$
k_{s c}=\sqrt{\frac{2}{\beta_{o}^{3} \gamma_{o}^{3}} \frac{I_{o}}{I_{A}} \frac{l^{2}}{a_{o}^{2}}} ; \quad k_{\beta}=\frac{\varepsilon l}{a_{o}^{2}} .
$$

In a periodic system comprised of $m$ bilaterally symmetric cells, ${ }^{8}$ such as in Fig. 2, evolution is determined by eigenvalues of the cell transport matrix $\mathbf{M}_{c}=\mathbf{M}(0 \mid T)$, $\mathbf{M}(0 \mid m T)=\mathbf{M}_{c}{ }^{m}[103]$ :

${ }^{7}$ Equations (25) are canonical with the following Hamiltonians:

$$
\hat{h}=\frac{{\widehat{a^{\prime}}}^{2}}{2}-k_{s c}^{2} \ln \hat{a}+\frac{k_{\beta}^{2}}{2 \hat{a}^{2}}=\frac{k_{\beta}^{2}}{2}=i n v ; \quad \tilde{h}(s)=\frac{\tilde{n}_{k}{ }^{2}}{2}+\frac{k_{\beta}^{2}}{\hat{a}^{2}(s)} \tilde{n}_{k}^{2} .
$$

${ }^{8}$ Bilateral symmetry of the cell provides for equality of the diagonal elements of $\mathbf{M}_{c}: m_{11}=m_{22}$.

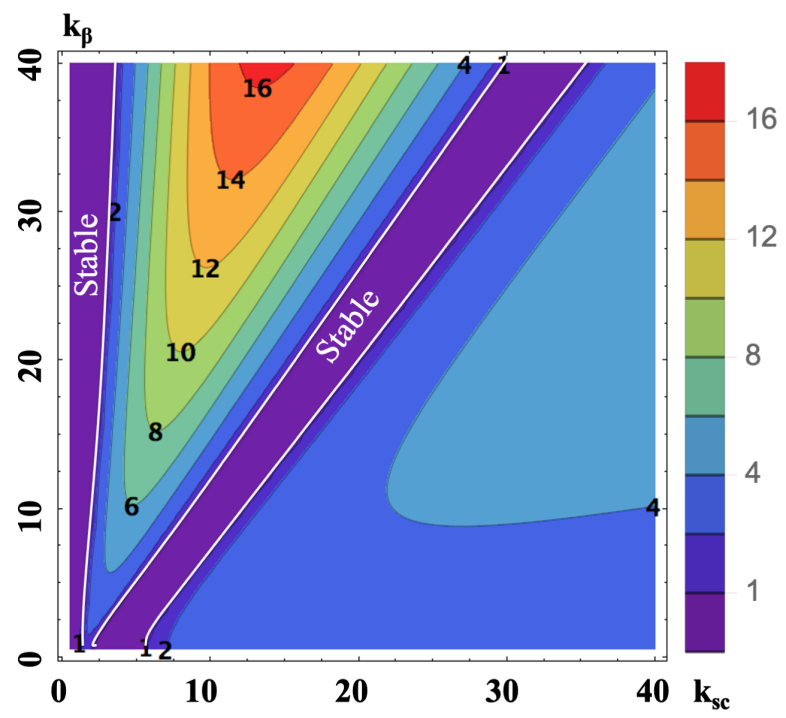

FIG. 4. Contour plots of the absolute value of the cell's growth rate: $\lambda=\max \left(\left|\operatorname{Re} \lambda_{1}\right|,\left|\operatorname{Re} \lambda_{2}\right|\right)$. The purple area highlighted by white lines shows the areas of the stable oscillations $\left|\lambda_{1,2}\right|=1$. Outside of these areas, the oscillations grow exponentially.

$$
\begin{aligned}
\mathbf{M}_{c} & =\left[\begin{array}{ll}
m_{11} & m_{12} \\
m_{21} & m_{11}
\end{array}\right] ; \quad \lambda_{1}=\lambda_{2}^{-1}=m_{11}-\sqrt{m_{11}^{2}-1} ; \\
\tilde{g}_{k}(m T) & =\frac{\lambda_{1}^{m}+\lambda_{1}^{-m}}{2} \tilde{g}_{k}(0)-\frac{m_{12}}{\sqrt{m_{11}^{2}-1}} \frac{\lambda_{1}^{m}-\lambda_{1}^{-m}}{2} \dot{\tilde{g}}_{k}(0),
\end{aligned}
$$

with $\left|m_{11}\right|>1$ indicating exponential growth, e.g., instability. With $\left|\lambda_{1}\right|^{m} \gg 1$, the amplitude grows asymptotically as $\lambda_{1}^{m} / 2$.

We evaluated the growth rate per cell in such a system with a semianalytical code in Mathematica [116] using the fourth order symplectic integrators [117] as a function of these two parameters. The results are shown in Figs. 3 and 4. Analysis shows that the growth rates peak along the ridge $k_{\beta} \approx 3 \cdot\left(k_{s c}-1.2\right)$ and can be estimated there as $\lambda \propto 1.25 k_{s c} \approx 1.5+0.413 k_{\beta}$. It is worth mentioning that the emittance of the beam, expressed through the value of $k_{\beta}$, plays an important role in this instability-the space charge alone (e.g., $k_{\beta}=0 ; k_{s c} \neq 0$ ) does not generate significant growth rates.

Equations (25)-(27) are derived for large wave numbers $k,{ }^{9} k a \gg 1$ which result in $k$-independent values of $\lambda$. Landau damping (24) naturally damps the instability at $k>k_{\max }$,

$$
k_{\max }=\frac{\ln \lambda}{T \sigma_{v}} ; \quad \omega_{\max }=\frac{v_{o}}{2 l} \cdot \frac{\gamma_{o}{ }^{3}}{\sigma_{\gamma}} \ln \lambda,
$$

where $\sigma_{\gamma} / \gamma_{o}$ is the relative energy spread in the beam: $\sigma_{\gamma} / \gamma_{o}=\sigma_{v} / c$ and $T$ is the propagation time. The energy

\footnotetext{
${ }^{9}$ Corresponding frequency of modulation in the lab frame is $\omega_{m}=\gamma_{o} v_{o} k$.
} 
spread should take into account angular spread in the beam, which changes the longitudinal velocity of particles.

The PCI growth is also inhibited at low $k$; where at $k \leq k_{\min }, k_{\min }\langle a(s)\rangle=1$, the period of the density modulation in the comoving frame is comparable with the beam's transverse size and the use of a simplified expression for the electric field (22) is no longer valid. In fact, detailed studies of the modulation growth at low $k$ (see $[75,118]$ ) provide an approximate scaling of the space charge coefficient at $k\langle a(s)\rangle<1: k_{s c} \rightarrow k^{*}{ }_{s c}=k_{s c} \cdot[k\langle a(s)\rangle]$. The PCI vanishes at long modulation periods (low $k$ ) when the "working point" $k^{*}{ }_{s c}, k_{\beta}$ reaches the stable region. Since real beams neither have transverse KV nor do they have $\kappa-1$ energy distributions, these theoretical results only can be used as a guide; while, when possible, the PCI should be evaluated numerically for real beam distributions in a real lattice using numerical codes (as explained in the next sections).

\section{B. General 3D case}

In the general case, the PCI can develop in beams experiencing complex dynamics, including but not limited to acceleration (or deceleration), energy chirping, bunch compression (or decompression), and emittance exchange. Beams can also be asymmetric and density perturbation may have both longitudinal and transverse components. There is a large variety of effects which can affect the development of PCI and coupling between longitudinal and transverse degrees of freedom.

For example, longitudinal bunch compression will result in a change of the longitudinal wave number $k$ proportionally to the increase of peak current. Similarly, transverse focusing will change the corresponding transverse wave numbers inversely proportionally to the beam sizes. Furthermore, acceleration (or deceleration) of the beam violates the assumption that the comoving frame is inertial and makes it, generally speaking, useless. It means that PCI theory can be properly developed only in the laboratory frame, with a number of important assumptions to be analytically manageable (see [99]).

The most general 3D case requires knowledge of the self-consistent evolution of the beam described by the linear $6 \times 6$ symplectic transport matrix $[99,104,119]$ :

$$
\begin{aligned}
X(t) & =\mathbf{M}(t) X(0) ; \quad \mathbf{M}(0)=\mathbf{I}_{6 \times 6} ; \quad \mathbf{M}^{T} \mathbf{S M}=\mathbf{M S M}^{T}=\mathbf{S} ; \\
\mathbf{S} & =\left[\begin{array}{cc}
\mathbf{0} & \mathbf{I}_{3 \times 3} \\
-\mathbf{I}_{3 \times 3} & \mathbf{0}
\end{array}\right] \rightarrow \operatorname{det} \mathbf{M}=1 ; \quad \mathbf{M}^{-1}=-\mathbf{S M}^{T} \mathbf{S} ; \\
\mathbf{M} & =\left[\begin{array}{cc}
\mathbf{A} & \mathbf{B} \\
\mathbf{C} & \mathbf{D}
\end{array}\right] ; \quad \mathbf{M}^{-1}=-\mathbf{S} \mathbf{M}^{T} \mathbf{S}=\left[\begin{array}{cc}
\mathbf{D}^{T} & -\mathbf{B}^{T} \\
-\mathbf{C}^{T} & \mathbf{A}^{T}
\end{array}\right],
\end{aligned}
$$

where $\mathbf{I}_{n \times n}$ is an $n$ by $n$ unit matrix, and we are using the phase space metric introduced by Alex Dragt [120], which provides for the convenient separation of coordinates $(x, y$, $z$ ) and canonical momenta $\left(P_{x}, P_{y}, P_{z}\right)$ :

$$
X^{T}=\left\{x, y, z, P_{x}, P_{y}, P_{z}\right\},
$$

where all six components in Eq. (30) are taken as a difference from that of a reference particle located close to the center of the beam. Detailed studies [99] show that linearized Vlasov-Maxwell equations can be written in the lab frame for harmonics of the density modulation with $\mathrm{k}$ vector, $\vec{k}_{\text {lab }}$, satisfying the following criteria:

$$
\begin{gathered}
\vec{k}_{\mathrm{lab}}=\vec{k}_{\perp}+\hat{z} k_{/ /} ; \quad k_{\mathrm{eff}}=\sqrt{\vec{k}_{\perp}^{2}+\frac{k_{/ /}^{2}}{\gamma^{2}}} ; \\
k_{\mathrm{eff}} a_{x} \gg 1 ; \quad k_{\mathrm{eff}} a_{y} \gg 1 ; \quad \gamma k_{\mathrm{eff}} a_{z} \gg 1,
\end{gathered}
$$

where $a_{x, y, z}$ are typical scales of the beam density inhomogeneity in three directions. These conditions locally approximate the case of infinite beam with constant spatial density and we can derive an integral equation for the evolution of $3 \mathrm{D}$ periodic perturbations, $\tilde{\rho}_{\vec{k}^{\prime}}(t)$,

$$
\begin{aligned}
f(q, p, t) & =F_{o}[q \cdot \mathbf{D}(t)-p \cdot \mathbf{B}(t), t]+\tilde{f}(q, p, t) ; \\
\tilde{\rho}_{\vec{k}}(t) & =e \iint e^{-i \vec{k} \vec{q}} d q^{3} d p^{3} \tilde{f}(q, p, t) ; \\
q & =(x, y, z) ; \quad p=\left(P_{x}, P_{y}, P_{z}\right) ;
\end{aligned}
$$

in the form of a linear integral equation with the kernel defined by the elements of the transport matrix (29) and the beam's initial momentum distribution function $F_{o}(P)$ :

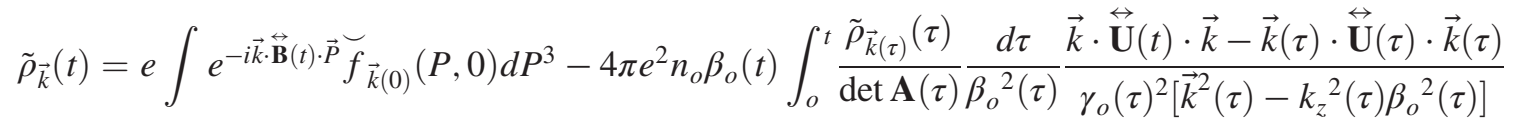

$$
\begin{aligned}
& \times \int e^{i(\vec{k}(\tau) \cdot \overleftrightarrow{\mathbf{B}}(\tau)-\vec{k} \cdot \overleftrightarrow{\mathbf{B}}(t)) \cdot \vec{P}} F_{o}(P) d P^{3},
\end{aligned}
$$

where $\mathbf{U}=\mathbf{U}^{T}=\mathbf{A}^{-1} \mathbf{B}^{T}$ and

$$
k \equiv\left\{k_{x}, k_{y}, k_{z}\right\} \equiv\left\{k_{1}, k_{2}, k_{3}\right\} ; \quad k(t)=k(0) \mathbf{A}^{-1}(t)
$$

describes the evolution of the $\mathrm{k}$ vector of the perturbation during the beam transport (e.g., compression, focusing, acceleration, etc.). It is worth noticing that in this case we did not make any further assumptions and the 
TABLE I. Electron beam parameters for PCI simulations and tests.

\begin{tabular}{lccc}
\hline \hline Name & Experiment & Case 1 & Case 2 \\
\hline Lattice & Aperiodic, & Periodic, & Periodic, \\
& LEBT & four cells & four cells \\
$\gamma$ & 3.45 & 28.5 & 275 \\
$\mathrm{E}, \mathrm{MeV}$ & 1.7 & 14.56 & 140.5 \\
$l, m$ & $1.5-3$ & 1 & 10 \\
$a_{0}, m m$ & $0.3, \mathrm{~min}$ & 0.2 & 0.1 \\
$I_{0}, \mathrm{~A}$ & 1.75 & 100 & 250 \\
$\varepsilon_{\text {norm }}, m$ & $1 \times 10^{-6}$ & $8 \times 10^{-6}$ & $4 \times 10^{-6}$ \\
$k_{s c}$ & Varies & 3.56 & 3.76 \\
$k_{\beta}$ & Varies & 7.02 & 14.55 \\
$\lambda_{1}$, per cell & $\mathrm{N} / \mathrm{A}$ & -4.06 & -5.10 \\
Energy spread & $1 \times 10^{-4}$ & $2 \times 10^{-4}$ & $1 \times 10^{-4}$ \\
Frequency, THz & 0.4 & 25 & 1,000 \\
\hline \hline
\end{tabular}

$F_{o}(P), P=\left.\left(P_{x}, P_{y}, P_{z}\right)\right|_{t=0}$ can be an arbitrary integrable function of momenta.

It is rather obvious that, with rare exception, the integral equation (33) has to be evaluated numerically. Nevertheless, a number of general observations can be derived from observing that the exponential term convoluted with the momentum distribution function is nothing else but the generalization of the Landau damping term that we discussed above.

First, it is well known that in most of the practical cases for real accelerators the longitudinal motion (compression, oscillations) is much slower than transverse ones. Usually, particles execute a number of transverse oscillations during a single bunch compression or decompression. In other words, the spread of transverse velocities results in multiple passing over typical sizes of inhomogeneity $a_{x, y}$. Thus, one can conclude that any transverse modulation at a scale smaller than $a_{x, y}$ will be washed away by Landau damping and will have no chance to grow. This means that transverse PCI represents predominantly academic interest, and this is why our paper focuses on longitudinal PCI.

More details about the integral equation (33) and a simplification for specific cases such as compression, acceleration, axial symmetry, etc., can be found in the main paper about PCI theory [99]. It also proves that under the same conditions and assumptions the integral equation (33) can be transformed into ordinary second order equation (23) for longitudinal PCI.

\section{NUMERICAL STUDIES}

While both qualitative and semianalytical studies expose the nature of the PCI, modern particle-in-cell codes allow 3D investigations of PCI without any predetermined assumptions. We used the code SPACE [121,122] for accurate simulation of PCI in an electron beam with a

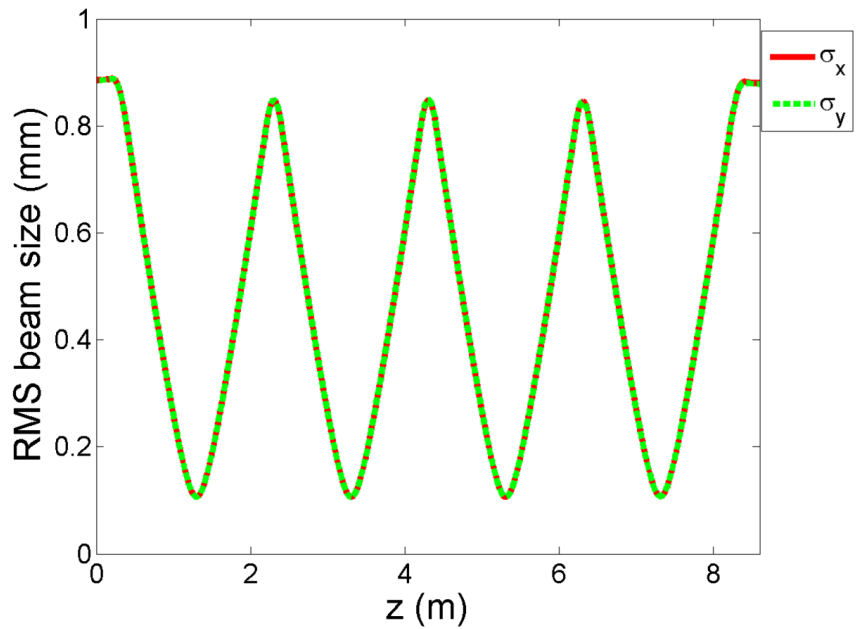

FIG. 5. Evolution of the transverse rms beam sizes in four cells of the PCI lattice of case 1: red-horizontal beam size; greenvertical beam size.

constant beam energy propagating along a straight section with focusing solenoids. Using this code, we confirmed that indeed the PCI could occur in periodic and aperiodic beam lines for a wide range of parameters, modulation frequencies and beam energies. Three sets of parameters listed in Table I were used in our studies.

Initial conditions for these simulations included shot noise and a weak longitudinal density modulation. The beam envelope at the entrance and the solenoid strengths are selected to provide the designed beam envelope with waists $a_{o}$ in the middle of each cell. The amplitude of the density modulation, $\tilde{\rho}_{k}$, is tracked as a function of the propagation distance.

For periodic lattices, each cell consists of a drift section with a length of $2 l$ between the two focusing solenoids. Figure 5 shows the self-consistent envelope of the electron beam propagating through the model four-cell periodic system with focusing solenoids (case 1), simulated by the code SPACE. Periodic transverse rms size variations from $0.85 \mathrm{~mm}$ in the solenoids to $0.1 \mathrm{~mm}$ in the beam waists result in strong PCI. Similar lattice-but tenfold longerwas used in the case 2 study. Evolution of the density perturbations at $25 \mathrm{THz}$ (wavelength of $12 \mu \mathrm{m}$ ) for case 1 and $1,000 \mathrm{THz}$ (wavelength of $0.3 \mu \mathrm{m}$ ) for case 2 is shown in Fig. 6. Evolution of the amplitude of the density modulation clearly demonstrates the nature of this instability; such that as plasma oscillations progress, the density modulation is transferred into the velocity modulation (at locations of the density minima), and then the velocity modulation generates an increased amplitude of density modulation, but with the opposite sign. This cascade-type growing plasma oscillation is the origin of the name for this instability-PCI.

Our 3D simulations confirmed expectations that $\mathrm{PCI}$ is a broadband instability, diminishing both at low and high frequencies. Figure 7 shows the amplitude gain spectrum of 
Density modulation, arb. units

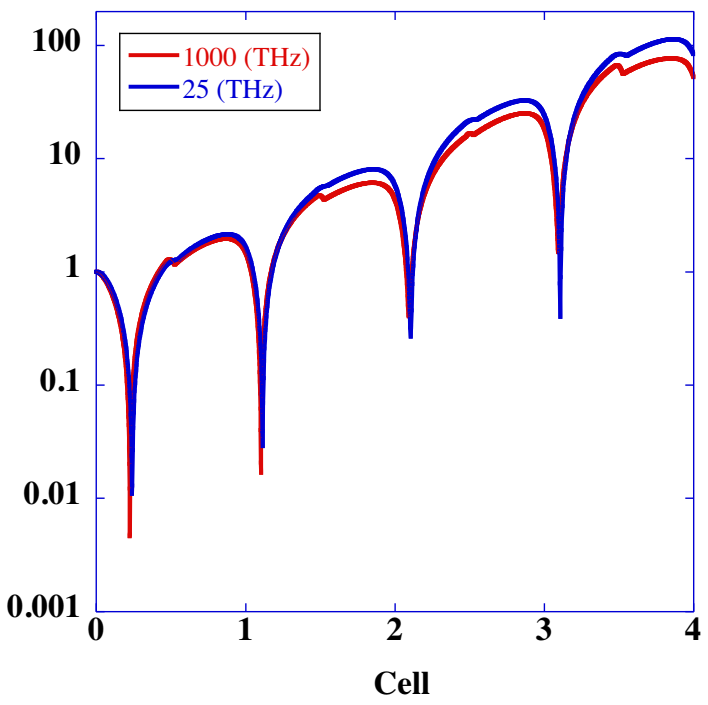

FIG. 6. Evolution of the amplitude of the density modulation amplitude in the four-cell PCI periodic lattice with parameters shown in Table I: blue line, case 1 , gain $=114$; red line, case 2 , gain $=75$.

the PCI microbunching instability in the four-cell periodic lattice (case 1) with bandwidth $\Delta f / f \sim 1$. It means that it can be used either as a technique for generating high power broadband $\mathrm{THz}$ radiation (with radiation power gain measured in tens of thousands) or used as a broadband amplifier (for example in a high energy hadron beam cooler [75]).

These simulations were performed in the linear PCI regime, with and without Poisson shot noise in the electron beam. Starting from the shot noise, PCI saturation would

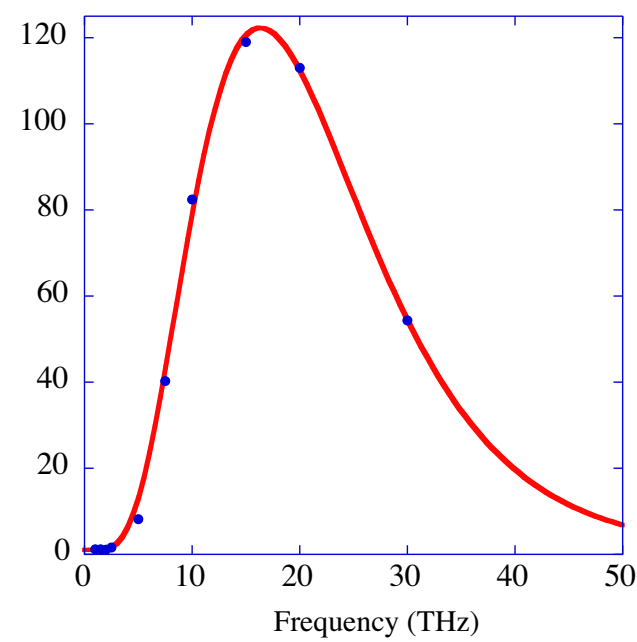

FIG. 7. Simulated PCI amplitude gain as a function of the modulation frequency: dots are results of simulation; the red curve is a fit. As expected from analytical studies, the PCI is a broadband microbunching instability with $\Delta f / f \sim 1$, but it is suppressed both at very low and very high frequencies. occur for case 1 and case 2 at the amplitude gains above 2,000 and 500, correspondingly.

Because of the exponential nature of the PCI, it is natural to compare increments of the instability. In case of periodic lattice, it is the growth rate per cell.

In our analytical model, based on $\kappa-1$ energy distribution,

$$
f_{o}(\gamma)=\frac{\sigma_{\gamma}}{\pi\left[\sigma_{\gamma}^{2}+\left(\gamma-\gamma_{o}\right)^{2}\right]}
$$

the growth rate per cell is the product of the eigenvalue $|\lambda|>1$ (27) and the Landau damping (24):

$$
\xi=|\lambda| L_{d \kappa 1} ; \quad L_{d \kappa 1}=\exp \left(-\frac{2 \omega_{o} l}{c\left(\beta_{o} \gamma_{o}\right)^{2}} \sigma_{\gamma}\right)
$$

Analytical estimations from beam parameters listed in Table I give the growth rate per cell, of $\xi_{1 a \kappa 1}=3.56$ for case 1 and $\xi_{2 a \kappa 1}=2.93$ for case 2 . It is worth noting that these estimations do not include reduction of the growth rate caused by finite transverse size of the electron beam and second order effects.

We performed limited number of 3D simulations using $\kappa-1$ energy distribution for case 1 . The sole purpose for this simulation was the comparison with our analytical estimates. The simulations predict growth rate of $\xi_{1 s \kappa 1}=$ 2.70 at frequency of $25 \mathrm{THz}$, e.g., $24 \%$ lower than the analytical estimate.

In most of our 3D numerical simulations we used Gaussian energy distribution, which models realistic beams better $^{10}$

$$
f_{o}(\gamma)=\frac{1}{\sqrt{2 \pi} \sigma_{\gamma}} e^{-\frac{\left(\gamma-\gamma_{o}\right)^{2}}{2 \sigma_{\gamma}^{2}}} .
$$

The pleasant surprise was that these simulations predict increments $\xi_{1 s G}=3.92$ for case 1 and $\xi_{2 s G}=3.78$ for case 2, which are slightly higher (by $10 \%$ and $29 \%$, correspondingly) than the analytical estimates for $\kappa-1$ energy distribution. The explanation lies in the fact that the $\kappa-1$ distribution is significantly wider than the Gaussian with the same $\sigma_{\gamma}$ : it has peak value $25 \%$ lower than the Gaussian and it also has longer tails. The Landau damping per cell can be estimated for Gaussian distribution as

$$
L_{d G}=\exp \left[-\frac{1}{2}\left(\frac{2 \omega_{o} l}{c\left(\beta_{o} \gamma_{o}\right)^{2}} \sigma_{\gamma}\right)^{2}\right]
$$

Using this Landau damping factor for analytical estimation would result in $\xi_{1 a G}=4.45$ for case 1 and $\xi_{2 a \kappa 1}=4.03$

\footnotetext{
${ }^{10} \mathrm{We}$ found that for a beam with Gaussian energy distribution it is impossible to reduce density evolution to a differential equation for analytical studies [99]. Hence, we used $\kappa-1$ energy distribution for analytical studies.
} 

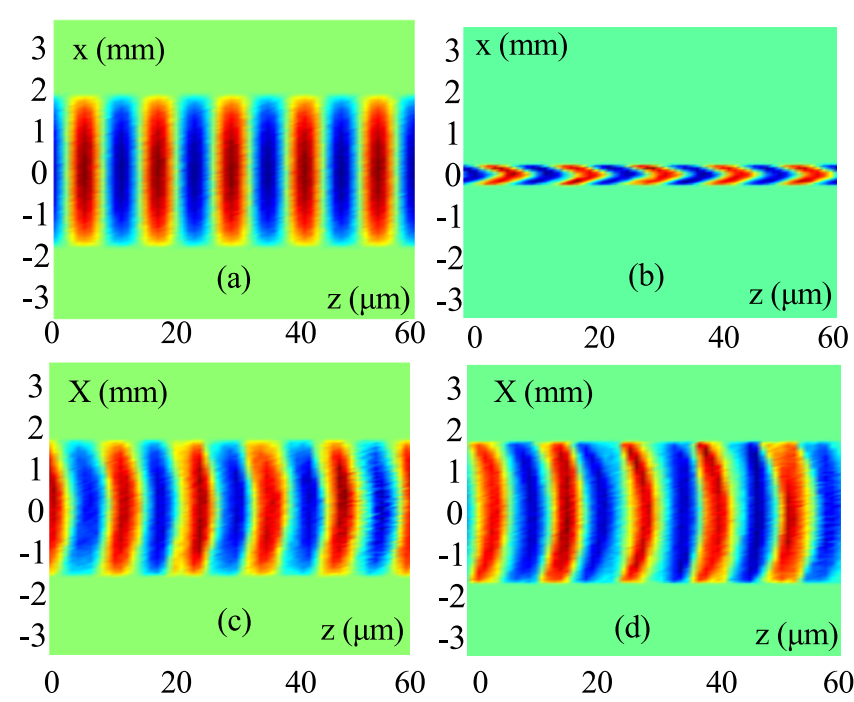

FIG. 8. Projection on the $\mathrm{z}$-x plane of the evolution of the $25 \mathrm{THz}$ periodic e-beam density modulation (case 1 in Table I). The background density is subtracted, and only variation of the density (ac portion) is shown: the red color corresponds to increased beam density and the blue to reduced beam density. Color density is normalized to maximum values to accommodate its exponential growth. (a) At the system entrance; (b) in the middle of the second cell (beam waist); (c) after the third cell, and (d) after the four-cell system. The electron beam is azimuthally symmetric: in the figures above, $z$ is the longitudinal coordinate with respect to the beam center, while $x$ is one of the transverse coordinates.

for case 2, which are $14 \%$ and $7 \%$ higher than results of our simulations.

Hence, we concluded that our analytical model works reasonably well for estimation of the PCI growth rate, but because of the exponential nature of the instability 3D selfconsistent simulations are required for accurate evaluation of PCI.

In contrast to the $1 \mathrm{D}$ analytical description, the 3D SPACE provides information about the spatial shape of the density modulation. Figure 8 illustrates such features as bending of the modulation wave fronts caused by the slowing down of electrons with large amplitude of transverse oscillation. ${ }^{11}$

3D evolution of a single spike in density modulation has much more complicated dynamics. Because of the charge conservation in the beam, such ac density perturbations have zero total charge. Figures 9-12 illustrate the appearance and disappearance of Langmuir waves [100,101] with a variety of transverse profiles. As a result of the competition, the main mode with maximum increment dominates at the exit of the four-cell structure.

After each cell, the structure resembling the initial density modulation reappears, albeit with the inverted sign

\footnotetext{
${ }^{11}$ Longitudinal velocity of a particle traveling with angle $\theta$ with respect to the beam direction is reduced as $\mathbf{v}_{s}=\mathbf{v} /\left(1+\theta^{2} / 2\right)$, where $\mathbf{v}$ is the total velocity of the particle.
}

and larger amplitude of the density modulation. In addition to amplification of the perturbation (the "color scale" changed from $10^{9}$ to $10^{11}$ ), the modulation could acquire a slight wave-front curvature. Our simulations show that the sign of the curvature can be either positive or negative depending on details of the lattice and beam parameters.

While periodic lattices are useful for comparing with theory or for developing a microbunching amplifier $[75,123,124]$, it was important to demonstrate the PCI experimentally and to compare experimental results with our simulation. The case of the aperiodic lattice is related to our experiment, which is described in the next section. The aperiodic lattice is nothing else but the low-energy beam transport line between the superconducting radio-frequency (SRF) gun and the SRF linac in the $\mathrm{CeC}$ accelerator comprising six focusing solenoids [125]—we used this system for our experimental demonstration of the plasmacascade instability.

The linear accelerator, shown in Fig. 13, was built for the coherent electron cooling $(\mathrm{CeC})$. The $\mathrm{CeC}$ accelerator has a ten-meter-long straight section, called low-energy beam transport (LEBT) with six nonequally spaced solenoids to propagate the electron beam, with full energy of $1.7 \mathrm{MeV}$, from the SRF photoemission electron gun to the SRF linac. According to our simulations, shown in Fig. 14, this lattice could excite very strong PCI at sub-THz frequencies in the electron beams with modest peak current $\sim 2 \mathrm{~A}$.

From our simulation and theoretical estimations, we get indication that PCI gain could be sufficiently high to reach saturation of this instability resulting in a measurable degree of density modulation. A high degree of density modulation then could be observed by using a timeresolving diagnostic system comprised of the SRF linac, the 45-degree dipole and the YAG4 profile monitor-the topic of the next section.

\section{EXPERIMENTAL PCI DEMONSTRATION}

We experimentally observed density modulation caused by the broadband PCI at frequencies $\sim 0.5 \mathrm{THz}$ while using the strong focusing lattice with beam envelopes shown in Fig. 14(a). The instability vanished when we used relaxed lattice resulting in a smooth beam envelope.

For this experimental demonstration we used 375 psec electron bunches with various charges generated in the 1.25 MV SRF photocathode gun. Two room temperature $500 \mathrm{MHz}$ rf cavities were used to correct the gun's rf curvature and reduce the relative energy spread in the bunch to $\sim 0.01 \%$. A strong-focusing aperiodic lattice comprised of six solenoids in the LEBT provided beam an envelope with strong envelope modulation and very high PCI gain at frequencies $\sim 0.4 \mathrm{THz}$ (Fig. 14).

To observe the density modulation in the beam we transformed our linac and the 45-degree dipole beam line into a time-resolving system with sub-psec resolution. We operated the $704 \mathrm{MHz}$ SRF linac at zero crossing with low 

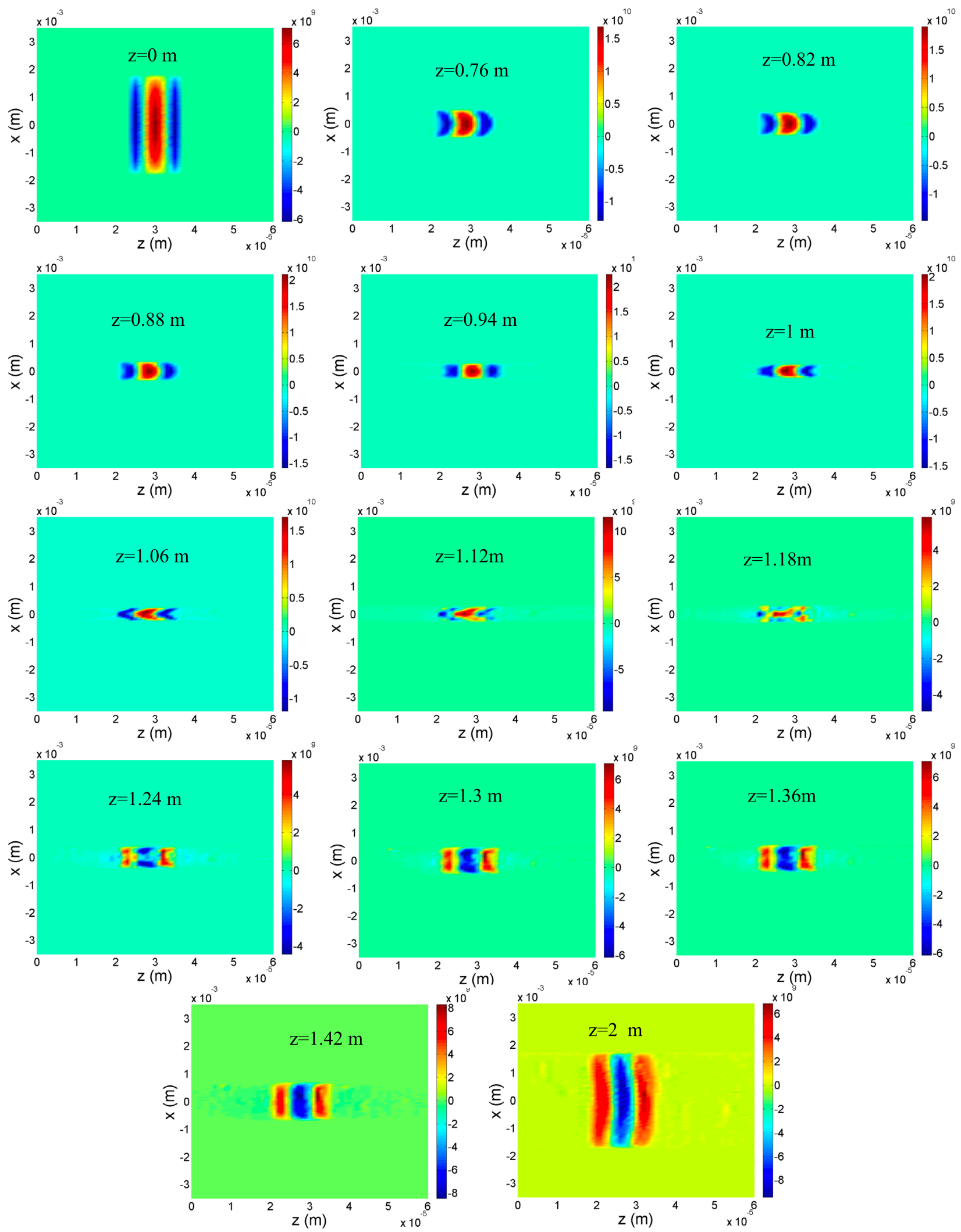

FIG. 9. Details of the single spike evolution in the first cell of the PCI lattice of case 1. Only the ac portion of the density is shown: red color corresponds to increased beam density and blue to reduced beam density with color density normalized to maximum values. Appearance of Langmuir waves with varying transverse structures can be seen in profiles taken from $z=1.12 \mathrm{~m}$ to $z=1.36 \mathrm{~m}$. Locations for taking profiles are chosen to illustrate the key processes occurring during the PCI process, including changes in the "wavefront" curvatures and in the transverse structure. 

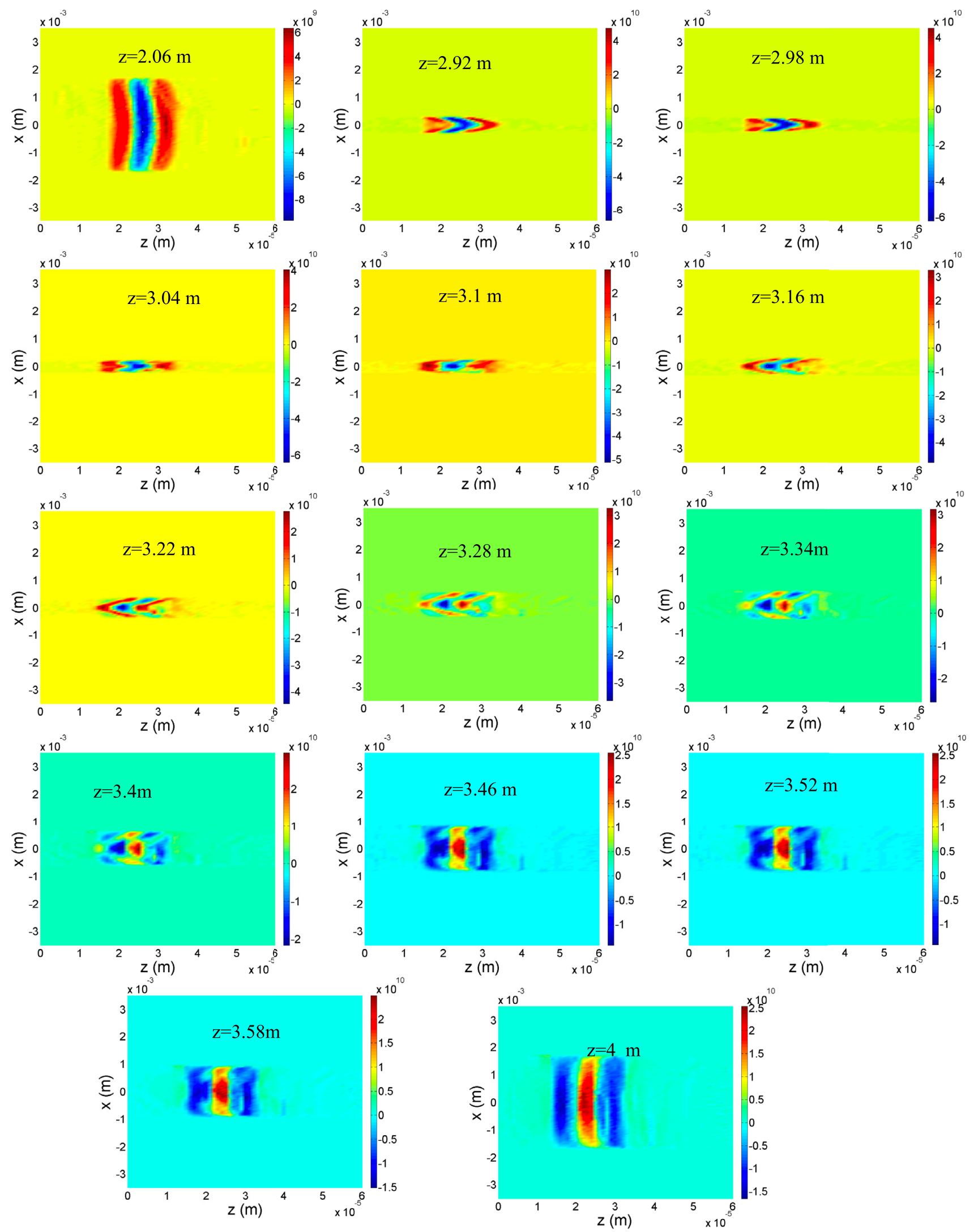

FIG. 10. Case 1: evolution of the single spike in density modulation in the second cell of the test PCI lattice. Two mode structures are visible at $z$ ranging from 3.3 to $3.5 \mathrm{~m}$. Note the reversal of the sign of the density modulation for the central slice, when compared with the first cell. 

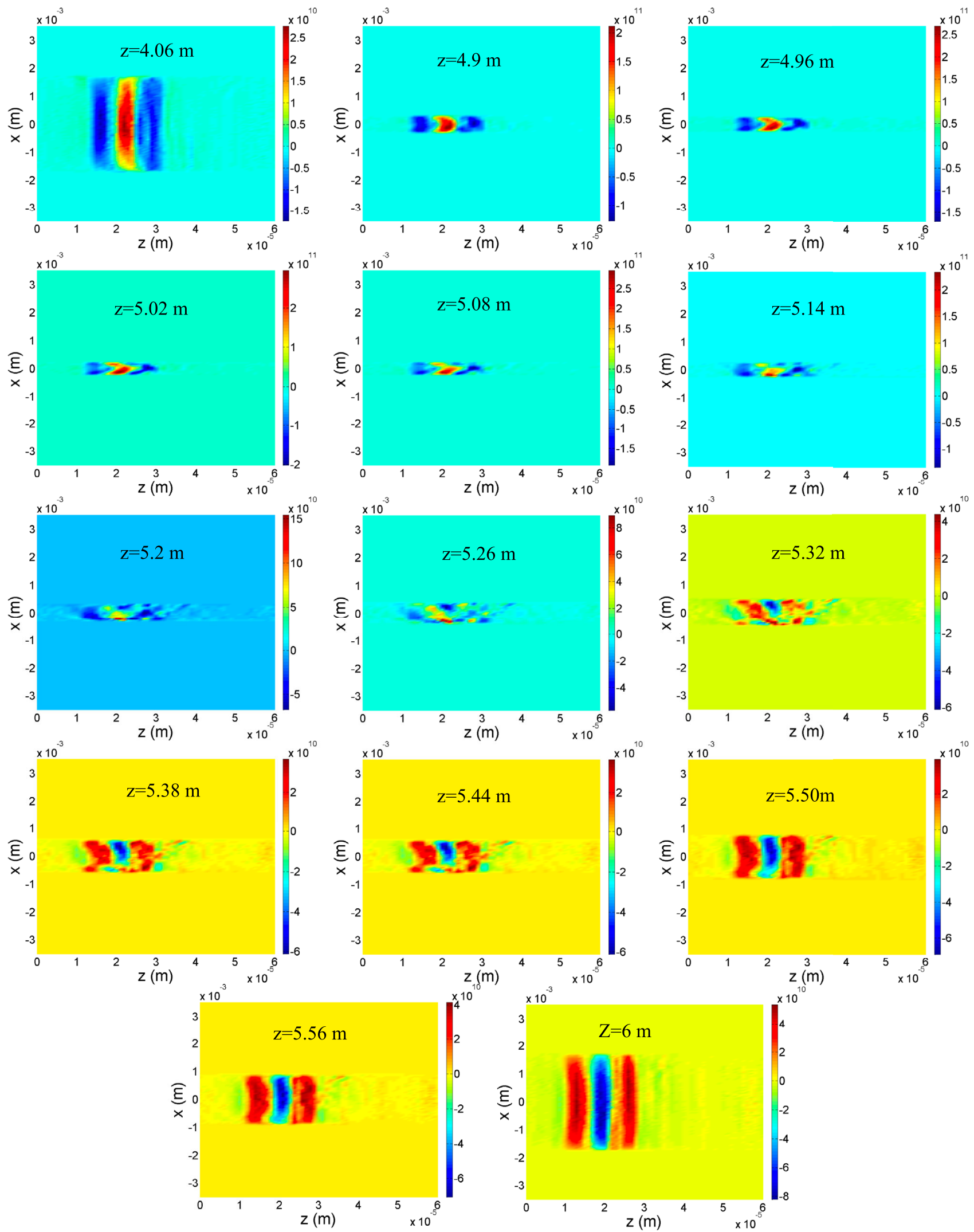

FIG. 11. Case 1: evolution of the single spike in density modulation in the third cell of the test PCI lattice. Fragmentation into multiple modes is visible at $z$ ranging from 5.05 to $5.5 \mathrm{~m}$. The sign of the density modulation for the central slice flipped again, and has the same sign as the first cell, but with tenfold larger amplitude. 

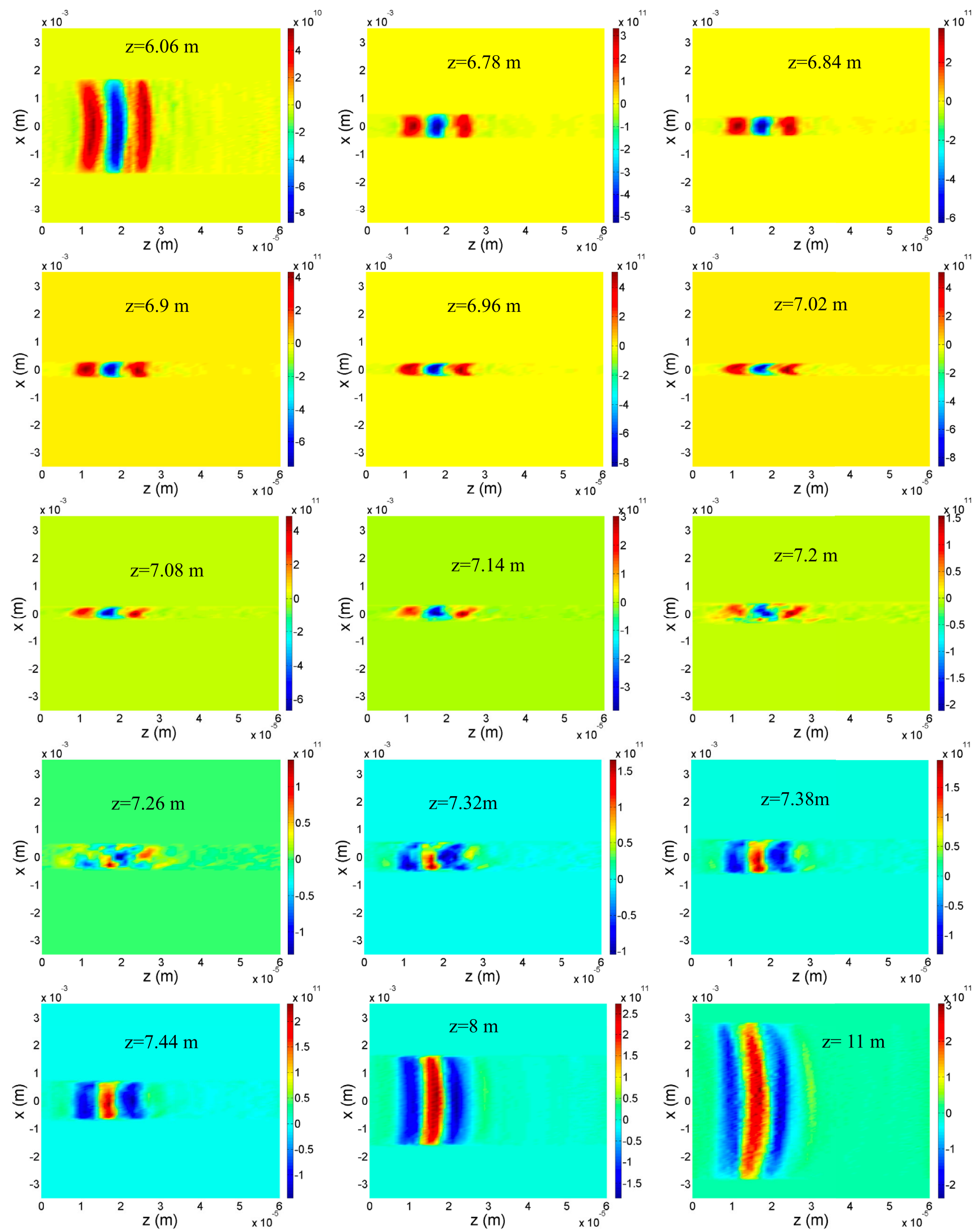

FIG. 12. Case 1: evolution of the single spike in density modulation in the fourth cell of the test PCI lattice (from $z=6$ to $z=8 \mathrm{~m}$ ). The beam was then propagated for 3 meters of free space resulting in well-defined structure shown in the last profile. Density modulation of the center slice has the same sign as at the entrance with 100-fold increase in the amplitude. 


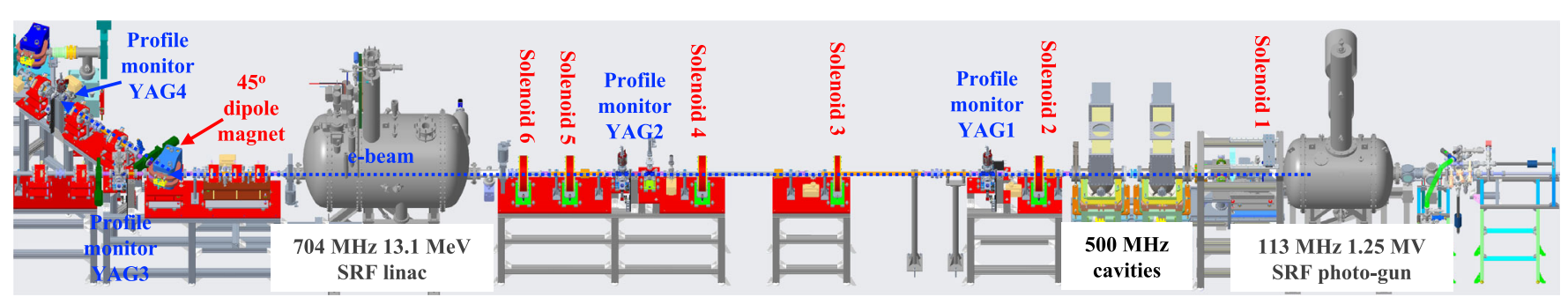

FIG. 13. Layout of the CeC accelerator (right to left): the SRF electron gun, two room-temperature rf cavities, the LEBT line equipped with six solenoids and two profile monitors, followed by the $13.1 \mathrm{MeV}$ SRF linac and a $45^{\circ}$ bending magnet beam line (with three quadrupoles and a beam profile monitor).

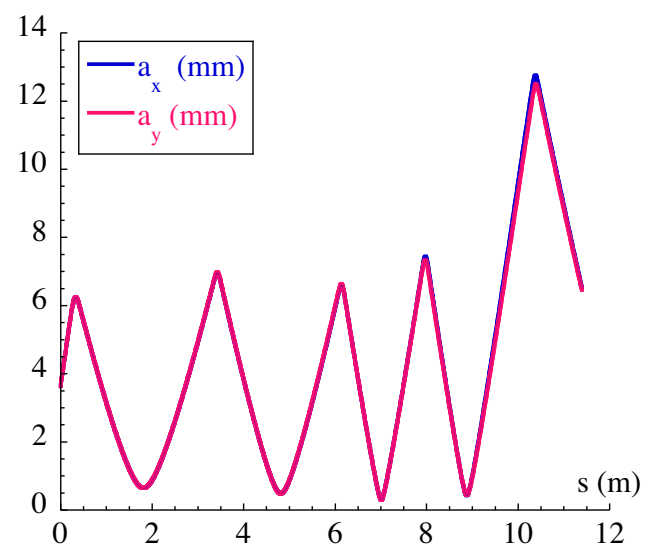

(a)

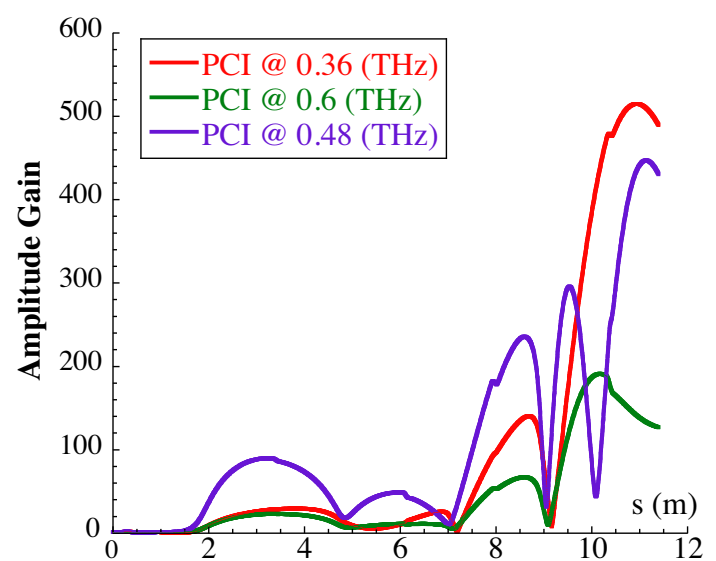

(b)

FIG. 14. (a) Simulated evolution of the horizontal and vertical beam envelopes and (b) PCI amplitude gain for modulation frequencies of $0.36 \mathrm{THz}$ (red line), $0.48 \mathrm{THz}$ (violet), and $0.6 \mathrm{THz}$ (green). Simulations were done with code SPACE for $1.7 \mathrm{MeV}(\gamma=3.45), 0.7 \mathrm{nC}$, $0.4 \mathrm{nsec}$ electron bunch with $1 \mu \mathrm{m}$ normalized slice emittance and $0.01 \%$ slice rms energy spread.

accelerating voltage, $V \sim 100-200 \mathrm{kV}$, to correlate the particle's energy with its arrival time,

$$
E=E_{o}+e V \sin \omega_{L} t,
$$

where $\omega_{L}=2 \pi f_{L}$ and $f_{L}=704 \mathrm{MHz}$. The $45^{\circ}$ dipole and the profile monitor YAG4 (see Fig. 13) served as the energy spectrometer. The measured energy distribution was a carbon copy of the bunch's longitudinal or time profile.

The temporal resolution of this measurement system depends on the linac voltage. We tested the system with voltages from 50 to $200 \mathrm{kV}$ and found that the best data quality was obtained at $100 \mathrm{kV}$; where, at lower voltages we did not have sufficient time resolution, but at higher voltages, diminishing electron bunch density was reducing the signal to noise ratio-see Fig. 15. The background noise from the digital camera was too high to use voltages above $200 \mathrm{kV}$, e.g., the signal to noise ratio would be too low for reliable data analysis.

Since density modulation grows from random shot noise, averaging images was not an option-it would erase the random modulation and generate useless smooth profiles. Hence, it was critical to measure time structure of a single bunch in a single shot. The $100-\mathrm{kV}$ setting provided $0.373 \mathrm{~mm} / \mathrm{psec}$ scaling at the profile monitor and, correspondingly, $7 \mathrm{pixel} / \mathrm{psec}$ at the digital camera. Combined with the profile monitor's YAG crystal spatial resolution $\sim 0.1 \mathrm{~mm}$, this provided for attainment of sub-psec time resolution-enabled by fine focusing using three quadrupoles in front of the dipole magnet.

Quadrupoles between the 45-degree dipole and the YAG4 profile monitor were turned off to inflate the horizontal dispersion to $D_{x}=1.36 \mathrm{~m}$. In this case the 1" $(25.4 \mathrm{~mm})$ YAG crystal was intercepting electrons with momentum spread of $\pm 0.93 \%$, corresponding to a $66 \mathrm{psec}$ time slice in the bunch center chirped by $100 \mathrm{kV}$ voltage in the linac. The $66 \mathrm{psec}$ slice corresponds to $\pm 8.3^{\circ}$ of the rf phase in the $704 \mathrm{MHz}$ SRF linac, which guarantees linearity of the "time to energy to horizontal position" scaling:

$$
\begin{aligned}
& \Delta x=D \cdot \frac{\Delta p}{p_{o}}=\frac{D}{1-\gamma_{o}^{-2}} \cdot \frac{\Delta \gamma}{\gamma_{o}} ; \quad \frac{\Delta \gamma}{\gamma_{o}}=\frac{\mathrm{eV} \sin \omega t}{E_{o}} \approx \frac{\mathrm{eV}}{E_{o}} \omega t ; \\
& \frac{\Delta x}{t}=\frac{D}{1-\gamma_{o}-^{2}} \cdot \frac{e V \omega}{E_{o}} \cong 0.37\left[\frac{\mathrm{mm}}{\mathrm{psec}}\right] .
\end{aligned}
$$



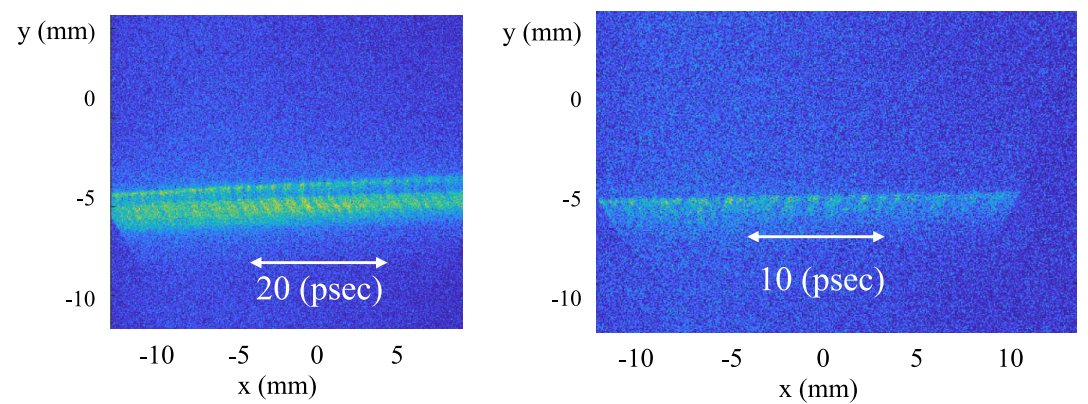

FIG. 15. Raw images from the 1" YAG screen taken with (a) $100 \mathrm{kV}$ and (b) $200 \mathrm{kV}$ voltage in the SRF linac using $0.7 \mathrm{nC}$ electron bunches. Three quadrupoles in front of the 45-degree dipole magnet were used to maximize the horizontal resolution. The measured horizontal dispersion of $D=1.36 \mathrm{~m}$ at the YAG4 profile monitor provides for 0.37 and $0.75 \mathrm{~mm} /$ psec resolution for 100 and $200 \mathrm{kV}$ linac voltages, respectively. The slice of electron beam seen at the screen corresponds to 65 psec in (a) and 32 psec in (b). Structures captured in these images are irregular with spectrum peaking around $0.5 \mathrm{THz}$.

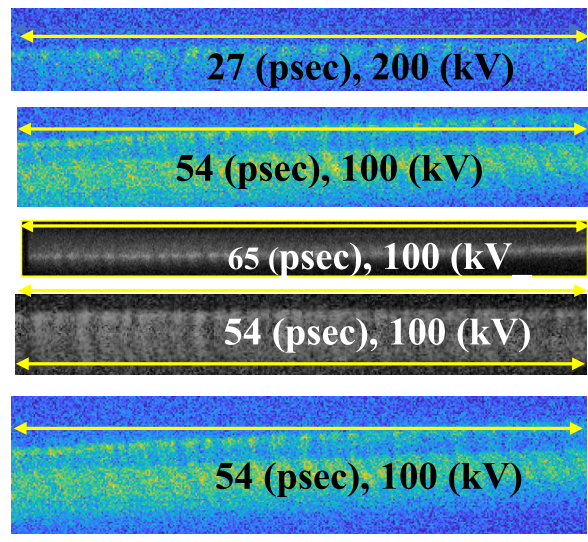

(a)

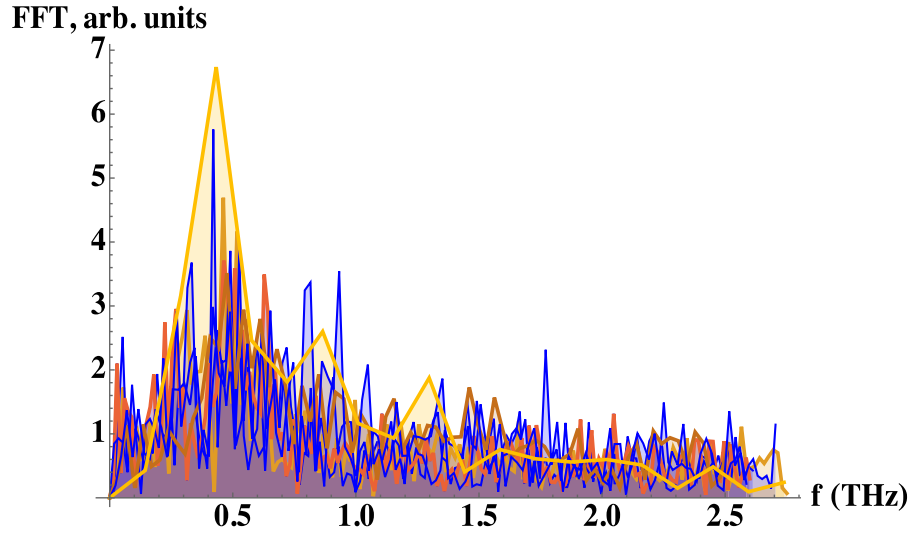

(b)

FIG. 16. (a) A subset of five YAG4 profile images showing distinct patterns of the density modulation driven by PCI in $0.7 \mathrm{nC}$ (peak current of $1.75 \mathrm{~A}$ ) electron bunches. (b) Twelve overlapped spectra of images taken for $0.7 \mathrm{nC}$ bunches. The noise floor above $1.5 \mathrm{THz}$ is determined by noise of the digital camera used for the imaging. The yellow curve shows a spectrum of density modulation extracted from the SPACE PCI simulations, where it has a maximum at about $0.45 \mathrm{THz}$ and secondary peaks at double and triple of this frequency as a sign of the PCI saturation.

The selected PCI lattice provided robust instability for a variety of bunch currents. We observed a strong modulation using bunch charges of 0.1 to $1 \mathrm{nC}$, for peak beam currents of 0.25 to 2.5 A. Maximum density modulation was observed using bunches with peak currents of 1 to $2 \mathrm{~A}$. Only at very low beam currents below $0.2 \mathrm{~A}$ was the instability no longer observable. As we mentioned above, use of a relaxed focusing lattice with smooth beam envelope eliminated the instability for all beam charges and currents.

Figure 16 displays a sample of five typical images taken with $0.7 \mathrm{nC}$ electron bunches as well as the spectra of the observed density modulation. Since density modulation grows from random shot noise, the spectrum of each individual sample is unique. Hence, we used the averaged spectrum to compare it with the simulations. As predicted by simulations, the peak of the density modulation spectra is located just below $0.5 \mathrm{THz}$ in full agreement with our simulation predicting the peak to be at $0.45 \mathrm{THz}$. Because of the saturation, there are secondary peaks at the double $(0.9 \mathrm{THz})$ and the triple $(1.35 \mathrm{THz})$ of the $0.45 \mathrm{THz}$ fundamental frequency. The measured spectrum is in good agreement with our simulations showing saturation of the PCI instability and a peak gain located at about $0.45 \mathrm{THz}$. Reduction of the PCI gain at low frequencies as well as high frequencies is also in good agreement with the SPACE simulations and expectation from the PCI theory. More details on measuring the density modulation can be found in Appendix B.

We carefully studied possible sources of density modulation with a special suspicion towards possible mode competition in the laser driver for our SRF photoemission electron gun. In fact, with an improperly tuned laser source it would be possible to observe modulation of the laser power with a frequency $\sim 50 \mathrm{GHz}$, which is easily observable in the electron beam as well. In order to avoid possible 


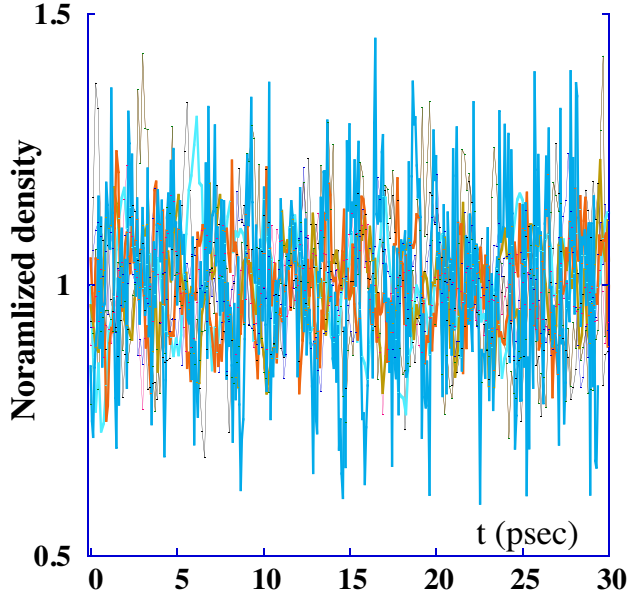

(a)

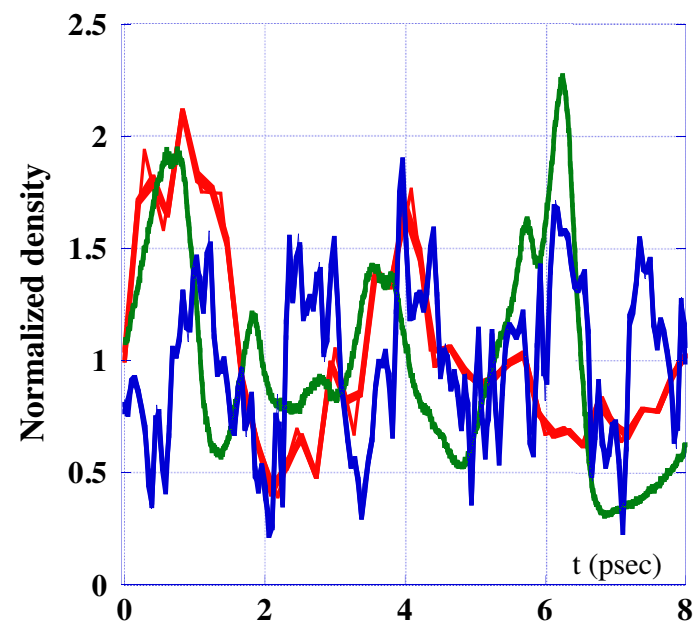

(b)

FIG. 17. (a) A 30-psec section of nine measured normalized density profiles. (b) An 8-psec section of three density profiles: SPACEcode simulation (green), measured with $100 \mathrm{kV}$ linac voltage (red) and $200 \mathrm{kV}$ linac voltage (blue).

sources of density modulation at frequencies of $\sim 0.5 \mathrm{THz}$, we measured the spectrum of the laser intensity and found that when improperly tuned it has FWHM bandwidth of $60 \mathrm{GHz}(0.06 \mathrm{THz})$ with diminishing power content above $100 \mathrm{GHz}$ - see Appendix $\mathrm{C}$ for details. We also tuned the laser to a single mode operation for the measurements shown in Fig. 16.

We also performed self-consistent simulations of electron beam dynamics in LEBT including wakefields of all elements in the LEBT and did not observe any instabilities at $\mathrm{THz}$ and sub-THz frequencies. Most importantly, these simulations clearly demonstrated that without PCI the longitudinal beam dynamics of the electron beam in the LEBT does not significantly depend on the transverse focusing and frequency spectrum of the bunch profile, as it contains only low $(\mathrm{GHz})$ frequencies for both PCI and relaxed LEBT lattices.

To clarify that the observed structures were indeed caused by the PCI we preformed the following tests: (a) We ran simulations of beam dynamics including and excluding wakefields from the beam-line components in LEBT (calculated using codes CST [126], ECHO [127], and $\mathrm{ABCI}$ [128]) and did not find any signature at frequencies above $0.01 \mathrm{THz}$. (b) We checked that modulation at frequencies $\sim 0.5 \mathrm{THz}$ does not exist in the laser pulses driving the beam. Moreover, the laser gain curve can accommodate only two modes separated by $0.05 \mathrm{THz}$. (c) We studied dependence of the density modulation on the electron beam charge. As predicted by simulation, there is a significant dependence of density modulation amplitude on the bunch charge. Instability disappears for charge per bunch below $100 \mathrm{pC}$. (d) Use of relaxed lattice with a smooth beam envelope eliminated observable THz-scale density modulation in the electron beam at all currents. This fact eliminates the possibility that observed density modulation can be the result of a conventional microbunching instability [79-97]—see detailed discussion in Appendix D.

The observed relative density modulations were very large: typically, at the levels $\pm 40 \%$ but occasionally reaching towards 100\%; which can be seen both in the captured images in Fig. 16(a) or in the density profiles shown in Fig. 17. While these profiles clearly show the random nature of the induced modulation, they also indicate that PCI was either saturated or was very close to saturation. While the profiles of the density modulation have a random nature, they exhibit similar time structure and similar depth of the density modulation predicted by our simulation, as shown in Fig. 17(b).

Hence, we concluded that the observed THz-scale density modulation was indeed the product of saturated plasma-cascade instability originating from a random shot noise in the electron beam.

Nominally, for $\mathrm{CeC}$ operations, we compress electron bunches 20 - to 30 -fold in the LEBT by applying an energy chirp by the $500 \mathrm{MHz}$ cavities. Such compression would transfer the $0.5 \mathrm{THz}$-scale modulation into that with frequencies $10 \mathrm{THz}$. Our simulations, using Impact-T [129] code showed (see Fig. 18) that using a strong focusing lattice in the LEBT would result in strong PCI at frequencies of about 10-15 THz. The simulations also showed that the use of relaxed lattice would suppress this PCI. These start-to-end self-consistent simulations were using the Impact-T code with 20 million microparticles, and running at the NERSC supercomputer [130]. The low frequency portion of the spectra represents that of the compressed electron bunch. At the same time, the noise floor at high frequencies was defined by the limitations from the code including the spikes-clearly seen in near 15 and $20 \mathrm{THz}$ for the relaxed lattice-being nothing else than the 


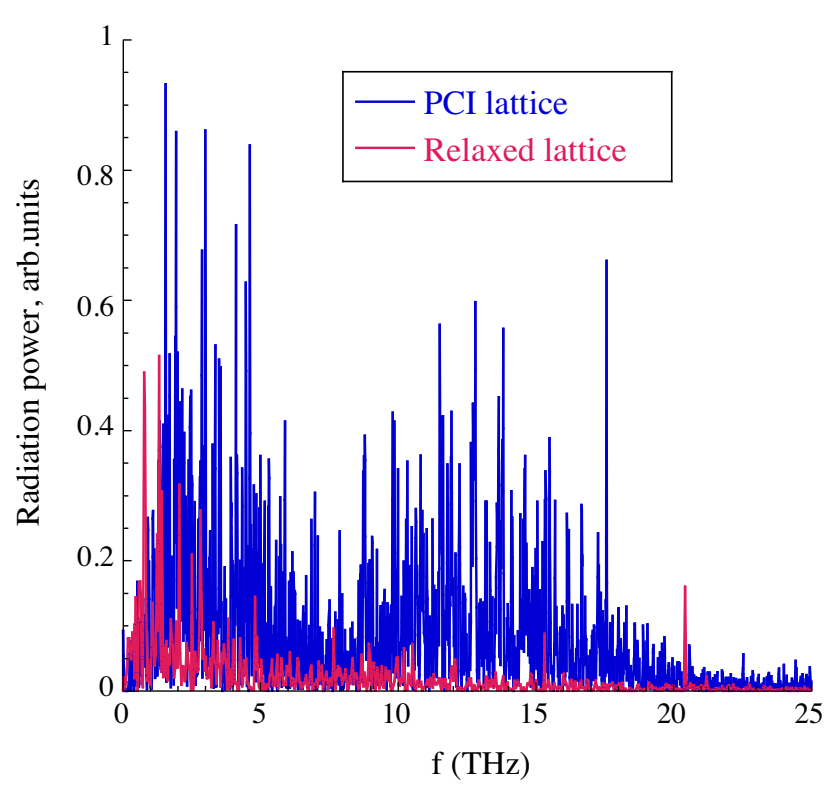

FIG. 18. Power spectra of the time distribution in the 1.5-nC electron bunch compressed to a peak current of $75 \mathrm{~A}$ and accelerated to $14.6 \mathrm{MeV}$ in the $\mathrm{CeC}$ accelerator, simulated by the Impact-T code. The blue line is for the beam propagating though a strong focusing PCI lattice, while the red line is for a beam propagating though the relaxed LEBT lattices. Strong focusing in the LEBT results in an increase of 1-2 orders of magnitude of the beam noise power at frequencies around $12.5 \mathrm{THz}$.

computing artifacts related to the finite mesh and the finite time steps.

The Impact-T simulations were the first successful predictions of the PCI in compressing and accelerating an electron beam.

To resolve structures at a $15-\mathrm{THz}$ scale, one would need a system with a resolution of $\sim 20 \mathrm{fsec}$, which were beyond capabilities of our system. Furthermore, our simulations did not predict PCI saturation with a density modulation of $\sim 100 \%$, which would further complicate direct observation of such density modulation.

Since direct observation of the density modulation was unmanageable, we made use of a measurement of the power of the far-IR radiation from the 45-degree dipole magnet as an indirect indication of the density modulation induced by PCI. Radiated power from the magnet was extracted by a cooper mirror, installed $0.7 \mathrm{~m}$ downstream of the radiation point, through a 1" diamond window. Extracted radiation was focused by an off-axis gold-coated parabolic mirror on the entrance window of the broadband IR Gentec-EO THZ-I-BNC pyroelectric detector. This detector has slow ac-coupled response (with a time constant of $\sim 50 \mathrm{msec}$ ) with a sensitivity of $2.12 \mathrm{mV} / \mathrm{nJ}$ to radiation at frequencies from 0.1 to $30 \mathrm{THz}$ (at wavelengths from $10 \mu \mathrm{m}-3 \mathrm{~mm}$ ). It has a noise equivalent of $0.6 \mathrm{nW} / \mathrm{Hz}^{1 / 2}$, which in our case corresponded to a noise floor of $\sim 100 \mathrm{pJ}$ per pulse. In order to cut the sub- $\mathrm{THz}$ radiation from affecting our power measurements, we installed a metalmesh screen at the exit window of the system. This mesh had a cutoff frequency of $\sim 1 \mathrm{THz}$.

We observed significant changes-by orders of magnitude-in the far-IR radiation power from the 14.6 MeV electron beam when we mismatched the electron beam envelope or when we increased the electron beam current. In both cases we used the so-called PCI lattice with a large modulation of the beam envelope. The peak current was controlled by the energy chirp provided by the 500-MHz bunching cavities.

We investigated dependencies of the far-IR power radiated by the electron beam on multiple parameters of the $\mathrm{CeC}$ accelerator with the goal of learning how to suppress such an instability in a real accelerator environment and how to control THz-scale noise in the electron beam. Results of two typical measurements are shown in Fig. 19.

When the beam is mismatched, and the beam envelope is undergoing oscillations in the LEBT lattice the microbunching PCI upsurges the density modulation and the corresponding far-IR radiation power of the electron beam. This behavior is illustrated Fig. 19(a). Specifically, the noise level is minimized at a solenoid current of about 3.7 A, but grows when focusing is too weak (e.g., at $2 \mathrm{~A}$ ) and the envelope is too large at the next solenoid, or when focusing is too strong (e.g., at solenoid current of $5 \mathrm{~A}$ ) and the beam is focused to a small spot downstream of the solenoid. Naturally, with weaker focusing, the beam (2 A setting) would have one less waist in the LEBT lattice when compared with overfocusing (5 A setting) and therefore PCI will have a lower gain.

An increase of the peak current by increasing the voltage in the bunching cavies, as shown in Fig. 19(b), results in an exponential growth of the radiation power in the PCI LEBT lattice. The increase of the radiation power by about 3,000 times corresponds to a growth of the amplitude of the density modulation by 35 -fold. If one considers each "waist" in the envelope-shown in Fig. 14(a) serving as the PCI cell, the average growth per cell would be $\sim 3.2$. Hence, the increase of the radiation power in this case can be considered as the result of the increase of the space charge coefficient, $k_{s c}$., on the PCI cell diagram in Fig. 4. In other words, the increase of the peak current $\left(k_{s c}\right)$ moves the operating point from the left to the right on the Fig. 4 diagram resulting in higher and higher gain per cell.

This part of our experimental program had more qualitative than quantitative aspects, when compared with the direct observation of the density modulation induced by PCI in the uncompressed electron bunch. Nevertheless, it provided us with two important improvements: (a) we indirectly proved that our prediction of PCA at frequencies $\sim 15 \mathrm{THz}$ were correct; (b) we demonstrated a practical method of controlling the strength of PCI and, when 


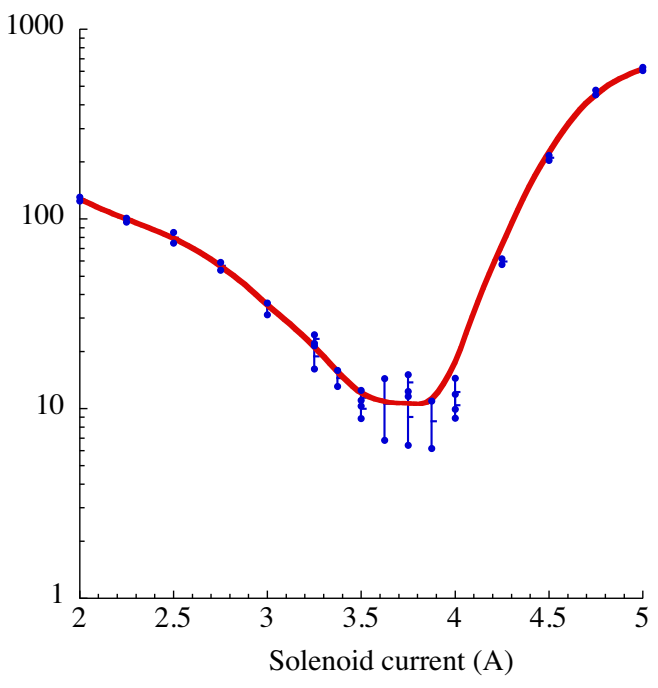

(a)

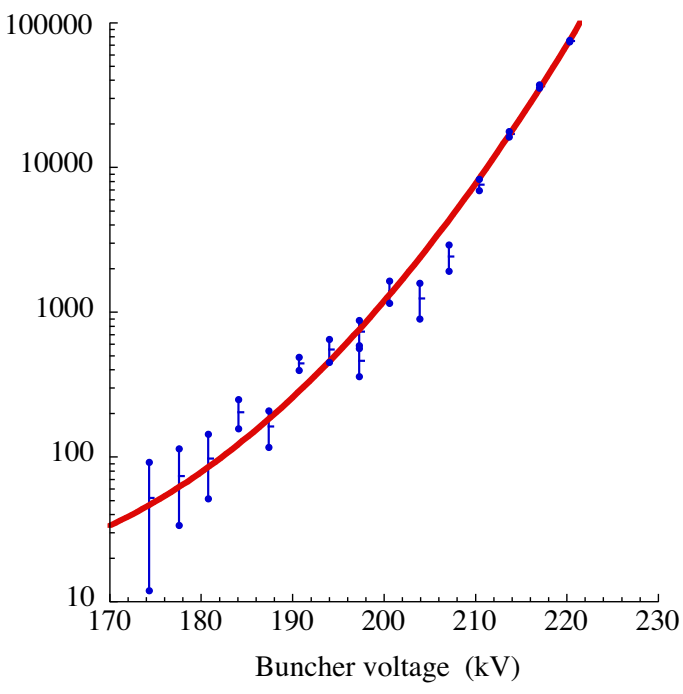

(b)

FIG. 19. Dependences of the IR radiation power from the 14.6-MeV electron beam with a 1.5-nC bunch charge as a function of (a) the current in one of the LEBT solenoids; (b) the voltage of the 500-MHz bunching cavities. Vertical axes for the radiation power have a logarithmic scale. The red continuous lines are smooth fits of the measured data. The radiation power is in units of the base-line value. ${ }^{12}$

necessary, suppressing it and reducing the noise in the electron beam to a desirable level.

\section{CONCLUSIONS AND ACKNOWLEDGMENTS}

In conclusion, we would like to announce the discovery of a novel microbunching instability occurring in charged particle beams propagating along a straight trajectory-the plasma-cascade instability. We first discovered the PCI theoretically, later investigated it in self-consistent simulations, and finally demonstrated it experimentally.

PCI can be both a menace and a blessing. It can strongly intensify longitudinal microbunching originating from the beam's shot noise or from an external source. Resulting random density and energy microstructures can degrade the beam quality. On the other hand, such broadband instability can drive novel high-power sources of radiation or can be used as a low-noise amplifier. For example, PCI can serve as a broadband amplifier in the $\mathrm{CeC}$ scheme [75] or can be used for boosting the power of $\mathrm{THz}$ and $\mathrm{PHz}$ radiation [76-78].

\section{ACKNOWLEDGMENTS}

The authors would like to thank all of their colleagues from $\mathrm{BNL}$ who contributed to the $\mathrm{CeC}$ project, with special acknowledgments going to Thomas Hayes, Geetha Narayan and Freddy Severino for their help with using CeC SRF linac for time resolved studies, Dr. Peter Thieberger for contributing to important 3D aspects of the

\footnotetext{
${ }^{12}$ We define the base-line power as that radiated by an electron beam with the same average current but with the shot-noise defined by Poisson statistics.
}

problem, and Dr. Thomas Roser for encouragement and unrelenting support of this research. The authors are thankful to Dr. Ji Qiang (LBNL) for his help with the Impact-T code leading to the successful simulation of $\mathrm{THz}$ $\mathrm{PCI}$ in the compressing and the accelerating $\mathrm{CeC}$ beam. The first author would also like to thank Professor Pietro Musumeci (UCLA), who mentioned during our discussion that the modulation of the transverse beam size can violate energy conservation in longitudinal plasma oscillations. This notion sparked the initial impulse for us to investigate if the modulation of the transverse beam size could cause a broadband longitudinal instability. This research was supported by NSF Grant No. PHY1415252, by DOE NP office Grant No. DE- FOA-0000632, and by Brookhaven Science Associates, LLC under Contract No. DE-SC0012704 with the U.S. Department of Energy.

\section{APPENDIX A: PARAMETRIZATION OF UNSTABLE MOTION}

The stability criteria and the parametrization of the 1D linear motion for the stable motion is well known, but it is generally not the case for the unstable motion. Here we briefly derive the necessary expression for this case using the Sylvester formula for matrix functions for a square matrix with distinct eigenvalues [131,132]. It is indeed the case for an unstable symplectic $2 \times 2$ matrix with a unit determinant:

$$
\begin{aligned}
\operatorname{det}\left[\mathbf{M}-\lambda_{1,2} \mathbf{I}\right] & =0 \Rightarrow \lambda_{1} \lambda_{2}=\operatorname{det} \mathbf{M}=1 ; \quad \lambda_{2}=\lambda_{1}{ }^{-1} ; \\
\max \left(\lambda_{1}, \lambda_{2}\right)>1 & \Rightarrow \lambda_{1} \neq \lambda_{2},
\end{aligned}
$$


which provides a simple expression for any analytical function of matrix M [131,132]:

$$
f(\mathbf{M})=f\left(\lambda_{1}\right) \frac{\mathbf{M}-\lambda_{2} \mathbf{I}}{\lambda_{1}-\lambda_{2}}+f\left(\lambda_{2}\right) \frac{\mathbf{M}-\lambda_{1} \mathbf{I}}{\lambda_{2}-\lambda_{1}},
$$

reducing the power of the matrix $\mathbf{M}$ to

$$
\mathbf{M}^{n}=\frac{\lambda_{1}{ }^{n}-\lambda_{2}{ }^{n}}{\lambda_{1}-\lambda_{2}} \mathbf{M}-\frac{\lambda_{1}^{n-1}-\lambda_{2}{ }^{n-1}}{\lambda_{1}-\lambda_{2}} \mathbf{I},
$$

where we took into account that $\lambda_{1} \lambda_{2}=1$. Hence elements of the matrix for $\boldsymbol{n}$ periods can be written as functions of elements of the matrix of the single period:

$$
\mathbf{M}^{n}=\frac{\lambda_{1}{ }^{n}-\lambda_{2}{ }^{n}}{\lambda_{1}-\lambda_{2}} \mathbf{M}-\frac{\lambda_{1}{ }^{n-1}-\lambda_{2}{ }^{n-1}}{\lambda_{1}-\lambda_{2}} \mathbf{I}=\left[\begin{array}{cc}
\frac{\lambda_{1}{ }^{n}-\lambda_{2}{ }^{n}}{\lambda_{1}-\lambda_{2}} m_{11}-\frac{\lambda_{1}{ }^{n-1}-\lambda_{2}{ }^{n-1}}{\lambda_{1}-\lambda_{2}} & \frac{\lambda_{1}{ }^{n}-\lambda_{2}{ }^{n}}{\lambda_{1}-\lambda_{2}} m_{12} \\
\frac{\lambda_{1}{ }^{n}-\lambda_{2}{ }^{n}}{\lambda_{1}-\lambda_{2}} m_{21} & \frac{\lambda_{1}{ }^{n}-\lambda_{2}{ }^{n}}{\lambda_{1}-\lambda_{2}} m_{22}-\frac{\lambda_{1}{ }^{n}-\lambda_{2}{ }^{n}}{\lambda_{1}-\lambda_{2}}
\end{array}\right]
$$

Using the fact that $\lambda_{1}+\lambda_{2}=\operatorname{Tr} \mathbf{M}=m_{11}+m_{22}$, by simple algebra (A5) can be reduced to

$$
\mathbf{M}^{n}=\left[\begin{array}{cc}
\frac{\lambda_{1}{ }^{n}+\lambda_{1}-n}{2}+\frac{m_{11}-m_{22}}{2} \frac{\lambda_{1}{ }^{n}-\lambda_{1}-n}{\lambda_{1}-\lambda_{1}{ }^{-1}} & m_{12} \frac{\lambda_{1}{ }^{n}-\lambda_{1}-n}{\lambda_{1}-\lambda_{1}{ }^{-1}} \\
m_{21} \frac{\lambda_{1}{ }^{n}-\lambda_{1}-n}{\lambda_{1}-\lambda_{1}{ }^{-1}} & \frac{\lambda_{1}{ }^{n}+\lambda_{1}{ }^{-n}}{2}-\frac{m_{11}-m_{22}}{2} \frac{\lambda_{1}{ }^{n}-\lambda_{1}-n}{\lambda_{1}-\lambda_{1}-1}
\end{array}\right]
$$

which is equivalent to Eq. (15).

For stable motion this parametrization is equivalent to that invented by Courant and Snyder for stable motion in periodical systems [103]. But in contrast to stable motion when both $m_{12}$ and $m_{21}$ elements cannot be equal to zero, in the case of unstable motion, this is allowed. Hence, introduction terms equivalent to $\beta$ and $\gamma$ functions could result in some of them having either zero or infinite values. Thus, (A6) gives the best parametrization for unstable matrices:

$$
\mathbf{M}^{n}=s n^{n}\left[\begin{array}{cc}
\cosh n \mu+s n^{n-1} \frac{m_{11}-m_{22}}{2 \sinh \mu} \sinh n \mu & s n^{n-1} \frac{m_{12}}{\sinh \mu} \sinh n \mu \\
s n^{n-1} \frac{m_{21}}{\sinh \mu} \sinh n \mu & \cosh n \mu-s n^{n-1} \frac{m_{11}-m_{22}}{2 \sinh \mu} \sinh n \mu
\end{array}\right] ; \quad \mu=\ln \left(\left|\lambda_{1}\right|\right) ; \quad s n=\frac{\lambda_{1}}{\left|\lambda_{1}\right|} .
$$

\section{APPENDIX B: TIME-RESOLVED MEASUREMENTS OF THE PCI-INDUCED MODULATION IN THE E-BEAM}

Our experimental setup was designed to measure time structure of the electron beam density but transferring the arrival time of particles into their energy deviation using the SRF linac at zero-crossing phase. Then we used the dogleg system with 45-degree dipole magnet as the beam energy spectrometer to convert the particle's energy into horizontal position on the beam profile monitor. We are using horizontal beam distribution on the profile monitor, which is not coupled with the vertical degree of freedom. Hence, we ignore motion in the vertical plane by taking slice at $y=$ const.
Measured distribution on the profile monitor is given by simple convolution of the beam's distribution function:

$\begin{aligned} \eta(x) & =\int \delta\left(x-x_{m}\right) f_{m}\left(X_{m}\right) d t_{m} d \gamma_{m} d x_{m} d x_{m}^{\prime} ; \\ X_{m}{ }^{T} & =\left[x_{m}, x_{m}^{\prime}, t_{m}, \gamma_{m}\right]\end{aligned}$

where we use subscript $m$ to indicate the measurement point. The accelerator section between the exit of the linac and the profile monitor had the dipole magnet as well as a set of quadrupoles. The beam central energy, $\gamma_{o}$, remains constant and the coordinates are transformed as follows: 


$$
\begin{aligned}
{\left[\begin{array}{c}
x_{m} \\
x_{m}^{\prime}
\end{array}\right] } & =M\left(s_{l} \mid s_{m}\right)\left[\begin{array}{l}
x_{l} \\
x_{l}^{\prime}
\end{array}\right]+\left[\begin{array}{c}
D\left(s_{m}\right) \\
D^{\prime}\left(s_{m}\right)
\end{array}\right] \delta_{b} ; \quad \delta=\frac{\gamma-\gamma_{o}}{\gamma_{o}} ; \\
t_{m} & =t_{m}\left(\gamma_{l}, t_{l}\right)+D^{\prime} x_{m}-D x_{m}^{\prime} ; \quad \gamma_{b, m}=\gamma_{b, m}\left(\gamma_{l}, t_{l}\right)
\end{aligned}
$$

where $D$ is horizontal dispersion at the profile monitor induced by the bending magnet (indicated by subscript ${ }_{b}$ ) and $M$ is the horizontal transport matrix for paraxial betatron motion. To account for possibility of evolving longitudinal instability after exit from the linac (indicated by subscript $_{l}$ ) we use a generic symplectic map in the longitudinal phase space. Finally, the linac introduces time dependent energy chirp:

$$
\gamma_{l}=\gamma+\gamma_{o} \Delta_{l} \cdot \sin \omega_{r f} t ; \quad \Delta_{l}=\frac{\mathrm{eV}_{r f}}{\gamma_{o} m c^{2}},
$$

where variables at the entrance into the linac do not have subscripts. The overall transformation from the entrance of the linac can be now written as

$$
\begin{aligned}
{\left[\begin{array}{c}
x_{m} \\
x_{m}^{\prime}
\end{array}\right] } & =M\left(s_{i} \mid s_{m}\right)\left[\begin{array}{c}
x \\
x^{\prime}
\end{array}\right]+\left[\begin{array}{c}
D\left(s_{m}\right) \\
D^{\prime}\left(s_{m}\right)
\end{array}\right] \delta_{b} ; \\
t_{m} & =\tau(\gamma, t)+D^{\prime} x_{m}-D x_{m}^{\prime} ; \\
\gamma_{b, m} & =\gamma_{b, m}\left(\gamma+\gamma_{o} \Delta_{l} \cdot \sin \omega_{r f} t, t\right) .
\end{aligned}
$$

The beam in the linear accelerator has zero dispersion and therefore the distribution function at the entrance of the linac can be factorized:

$$
f_{\text {in }}(X)=f_{\|}(\gamma, t) \cdot f_{\perp}\left(x, x^{\prime}\right) .
$$

Conservation of the phase-space density connects distribution function with the initial as [107]

$$
\begin{aligned}
f_{m}\left[X_{m}(X)\right] & =f_{i}(X) \Leftrightarrow f_{m}\left(x_{m}, x_{m}^{\prime}, t_{m}, \gamma_{m}\right) \\
& =f_{\|}(\gamma, t) \cdot f_{\perp}\left(x, x^{\prime}\right),
\end{aligned}
$$

which allows us to rewrite (B1) as

$\eta(x)=\int f_{\|}(\gamma, t) d t d \gamma \int \delta\left(x-x_{m}\right) \cdot f_{\perp}\left(x, x^{\prime}\right) d x d x^{\prime}$,

using simplicity of the $X \rightarrow X_{m}$ transfer map and related unity of the transformation's Jacobian.

For the next step, let us assume that transverse distribution at the entrance of the linac has standard Gaussian distribution using the well-known Courant-Snider invariant for the betatron motion [103]:

$$
f_{\perp}\left(x, x^{\prime}\right)=\frac{1}{2 \pi \varepsilon_{x}} e^{-I_{x}} ; \quad I_{x}=\frac{x^{2}+\left(\beta_{x} x^{\prime}+\alpha_{x} x\right)^{2}}{\beta_{x}}=\text { inv } .
$$

Using a standard accelerator physics technique for the second integral in (B7) we get

$$
\begin{aligned}
{\left[\begin{array}{c}
x_{m} \\
x_{m}^{\prime}
\end{array}\right] } & =\left[\begin{array}{l}
x_{\beta} \\
x_{\beta}^{\prime}
\end{array}\right]+\left[\begin{array}{l}
D \\
D^{\prime}
\end{array}\right] \delta_{b} ; \quad\left[\begin{array}{l}
x_{\beta} \\
x_{\beta}^{\prime}
\end{array}\right]=M\left[\begin{array}{l}
x \\
x^{\prime}
\end{array}\right] ; \\
M & =\left[\begin{array}{ll}
m_{11} & m_{12} \\
m_{21} & m_{22}
\end{array}\right] ; \\
I\left(x_{\beta}, x_{\beta}^{\prime}\right) & =\frac{x_{\beta}^{2}+\left[\beta\left(s_{m}\right) x_{\beta}^{\prime}+\alpha\left(s_{m}\right) x_{\beta}\right]^{2}}{2 \beta\left(s_{m}\right)} ; \\
& \frac{1}{2 \pi \varepsilon_{x}} \int \delta\left(x-x_{\beta}-D \delta_{b}\right) \cdot e^{-\frac{I\left(x_{\beta}, x_{\beta}^{\prime}\right)}{2 \varepsilon_{x}}} d x_{\beta} d x_{\beta}^{\prime}=e^{-\frac{\left(x-D \delta_{b}\right)}{2 \sigma_{x}^{2}}} \\
\sigma_{x}^{2}= & \beta\left(s_{m}\right) \varepsilon_{x},
\end{aligned}
$$

reducing $(\mathrm{B} 7)$ to

$$
\eta(x)=\int f_{\|}(\gamma, t) d t d \gamma e^{-\frac{\left(x-D \delta_{b}(\gamma, t)\right)^{2}}{2 \sigma_{x}^{2}}},
$$

with $\delta_{b}(\gamma, t)$ defining correlations between position on the profile monitor and particle location in the longitudinal phase space, and $\sigma_{\delta}=\sigma_{x} / D$ defining energy resolution of the system. Naturally, focusing electron beam in small spot is important for high resolution. We used triplet of quadrupoles between the linac and the dipole to optimize the resolution, but did not use focusing downstream of the dipole magnets to maximize value of the dispersion.

Equation (B9) states that measured distribution $\eta(x)$ depends only on the distribution function at the entrance of the linac, and evolution of the particle's energy between the entrance to the linac and the bending magnet. Specifically, Eq. (B9) states that modifications in the particle's arriving time, for example resulting from instability occurring between the linac and the profile monitor, do not play any role. In other words, Eq. (B9) is the result of causality and the fact that measured profile is an integral in time of the entire electron bunch.

At the same time, as can be seen from Eqs. (B4) and (B9), induced energy modulation could affect the profile. Let us assume that energy distribution also has Gaussian distribution with centroid dependent on the position in the bunch (slice):

$$
\begin{aligned}
& f_{\|}(\gamma, t)=f_{\|}(t) e^{-\frac{\left[\delta-\Delta_{o}(t)\right]^{2}}{2 \sigma_{\delta}^{2}}} ; \\
& \delta_{b}(\gamma, t)=\delta+\Delta_{l} \cdot \sin \omega_{r f} t+\Delta_{1}(t),
\end{aligned}
$$

with $\Delta_{o}(t)$ and $\Delta_{1}(t)$ being energy variations accumulated prior to the linac and between the linac and the energy spectrometer, correspondingly. Integrating over energy gives the final expression for the observed profile: 


$$
\begin{aligned}
& \eta(x)=\int d t \cdot f_{\|}(t) \cdot e^{-\frac{\left(\frac{x}{D}-\Delta_{l} \cdot \sin \omega_{r f} t+\Delta(t)\right)^{2}}{2 \sigma_{\Delta}^{2}}} ; \\
& \Delta(t)=\Delta_{o}(t)+\Delta_{1}(t) ; \quad \sigma_{\Delta}^{2}=\frac{\beta\left(s_{m}\right) \varepsilon_{x}}{D^{2}}+\sigma_{\delta}^{2} .
\end{aligned}
$$

For a portion of the bunch the energy change in the linac is linearized, $\sin \omega_{r f} t \cong \omega_{r f} t$, introducing linear chirp:

$$
\begin{gathered}
g=\Delta_{l}=\frac{e V_{r f} \omega_{r f}}{\gamma_{o} m c^{2}} . \\
\tau=t-\frac{x}{D g}+\frac{\Delta(t)}{g} ; \quad t=t(\tau) ; \\
\eta(x)=\int \frac{d \tau}{1+\frac{\Delta^{\prime}[t(\tau)]}{g}} \cdot f_{\|}\left(\frac{x}{D g}+\tau+\frac{\Delta[t(\tau)]}{g}\right) \cdot e^{-\frac{\tau^{2}}{2 \sigma_{\tau}^{2}}},
\end{gathered}
$$

with $\sigma_{\tau}$ indicating available time resolution of the system:

$$
\sigma_{\tau}=\frac{\sigma_{\Delta}}{g}=\frac{\gamma_{o} m c^{2}}{\mathrm{eV}_{r f} \omega_{r f}}\left(\frac{\beta\left(s_{m}\right) \varepsilon_{x}}{D^{2}}+\sigma_{\delta}^{2}\right) .
$$

In the case of weak energy chirp when $|g| \sim\left|\Delta^{\prime}\right|$, the correspondence between longitudinal beam profile and $\eta(x)$ on the profile monitor can be strongly distorted.

Since by definition $\langle\Delta(t)\rangle=0$, we can select $t=0$ at a location where $\Delta=0$. Then in the case of the strong chirp, $\left|\Delta^{\prime}\right| \ll|g|$, we also have $|\Delta(t)| \ll|g t|$ and

$$
\eta(x) \cong \int d t\left(1-\frac{\Delta^{\prime}(t)}{g}\right) f_{\|}\left(\frac{x}{D g}+t\right) \cdot e^{-\frac{t^{2}}{2 \sigma_{\tau}^{2}}},
$$

e.g., the energy modulation introduces additional modulation of $\Delta^{\prime}(t) / g$ on the profile monitor. This formula is especially useful, when energy perturbations are very small (see Appendix D).

In absence of energy modulation $(\Delta=0)$, the system will generate an image of the time structure in the incoming beam

$$
\eta_{p m}(x)=\text { const } \cdot \int f_{t}\left(\frac{x}{D g}+\tau\right) e^{-\frac{\tau^{2}}{2 \sigma \tau^{2}}} d \tau
$$

with time resolution of $\sigma_{\tau}$ and scaling between the horizontal position at the monitor and the time of $x=D g \cdot t$.

If beam does not have time structure, $f_{\|}(t)=$ const we get projection of the time-dependent energy modulation in the form of

$$
\eta(x)=\mathrm{const} \cdot \int d t e^{-\frac{\left(\frac{x}{D g}-t+\frac{\Delta(t)}{g}\right)^{2}}{2 \sigma_{\tau}^{2}}},
$$

which only can be evaluated for specific amplitude and shape of $\Delta(t)$. For cold beam we get

$$
\eta(x)=\frac{\text { const }}{g-\Delta^{\prime}\left(t^{*}\right)} ; \quad D\left[g t^{*}+\Delta\left(t^{*}\right)\right]=x,
$$

or in parametrric form

$$
\eta\{D[g t+\Delta(t)]\}=\frac{\text { const }}{g-\Delta^{\prime}(t)} .
$$

For this experiment we generated a single $1.76 \mathrm{MeV}$ electron bunch in a 113-MHz SRF gun with a flattop time profile having a FWHM duration of 375 psec with rise and the fall times of $\sim 105$ psec (see Appendix C, see Fig. 21(b)). We decelerated the beam by $64 \mathrm{kV}$ in the $500 \mathrm{MHz}$ cavities to compensate for the gun's voltage curvature and to generate a flat energy profile in the entire electron bunch with a remaining relative rms energy spread of $\sim 10^{-4}$. This monoenergetic beam was propagated thought the LEBT lattice to the 704-MHz SRF linac. The phase of the linac was carefully adjusted to have a zero crossing at the electron bunch center. This $100-\mathrm{kV}$ linac voltage created an $8.7 \%$ energy chirp from head to tail of the 375-psec bunch and corresponded to a 9.6\% energy change in the particle's momentum (rigidity). This energy chirp was doubled at a voltage of $200 \mathrm{kV}$.

The energy distribution in the electrons was measured using a YAG transverse beam profile monitor located 1.3 meters downstream of the 45-degree dipole magnet. Current in the dipole magnet was set to the central energy of the electron beam to observe the electron bunch distribution at the YAG screen. The measured horizontal dispersion function at this location was $D=1.36$ meter. Hence, a $\pm 0.93 \%$ portion of the beam momentum spread (e.g., $\pm 0.85 \%$ of the energy spread) was intercepted by the 1-inch-diameter YAG screen. This portion of the energy spectra corresponded to \pm 8.3 degrees of the linac phase for a voltage of $100 \mathrm{kV}$ (and, naturally, half of that for a voltage of $200 \mathrm{kV}$ ) where the energy and momentum chirps are practically linear functions of the arrival time. In fact, the deviation of $t->x$ from the linear scaling is less than $0.4 \%$ over the entire YAG image. Hence, we can use linear correlations between the horizontal position on the YAG screen and the arrival time of electrons to the linac entrance as

$$
t=\frac{x}{\omega_{r f} D} \cdot \frac{\gamma_{o} m c^{2}}{\mathrm{eV}_{r f}} ; \quad \gamma_{o}=3.32
$$

providing scaling of $2.57 \mathrm{psec} / \mathrm{mm}$ for $100-\mathrm{kV}$ and $1.28 \mathrm{psec} / \mathrm{mm}$ for $200-\mathrm{kV}$ voltages. This results in 7 and $14 \mathrm{pixel} / \mathrm{psec}$, respectively, at our digital CCD camera. With proper focusing of the electron beam, this resolution was sufficient to observe psec-scale density structures in the electron beam.

The challenges in these measurements were twofold: (i) the need to measure the structure in a single electron 


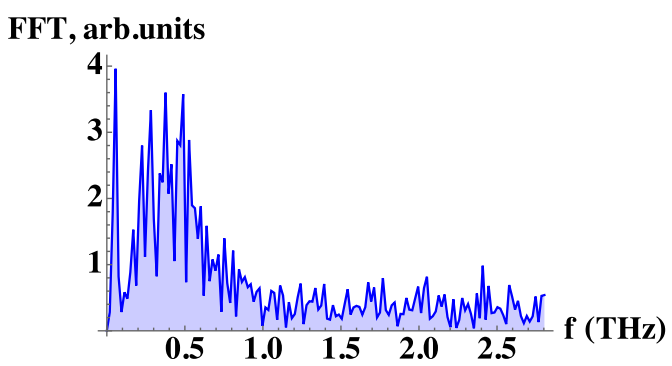

(a)

FFT, arb.units

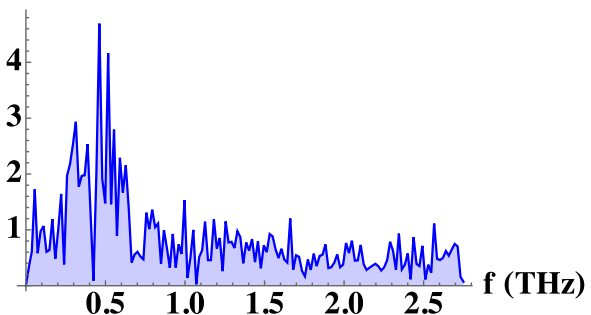

(c)

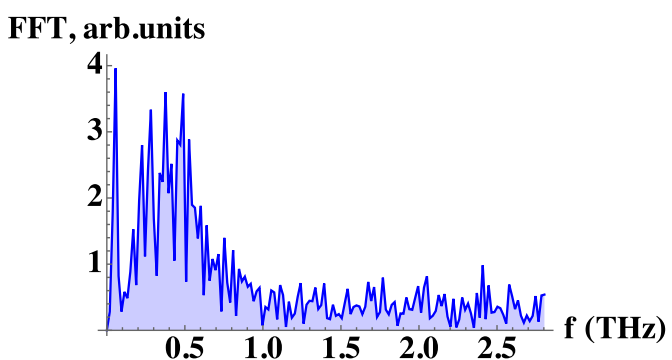

(e)

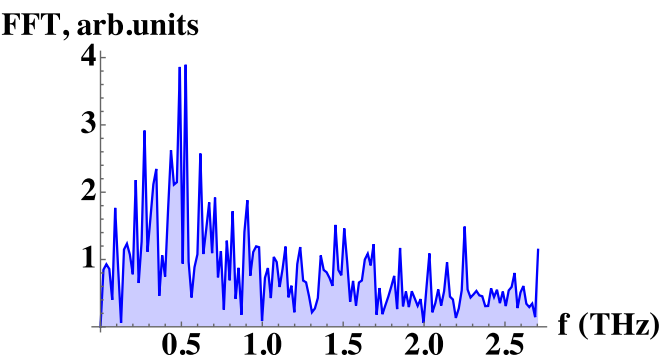

(b)

FFT, arb.units

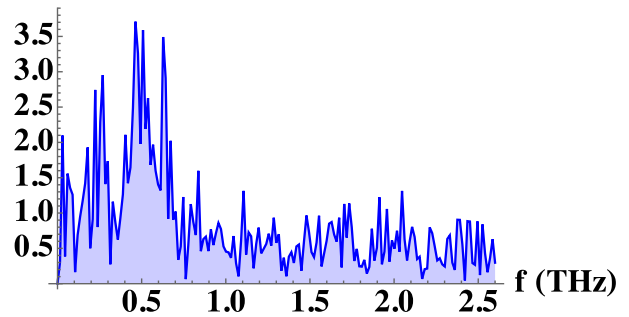

(d)

FFT, arb.units

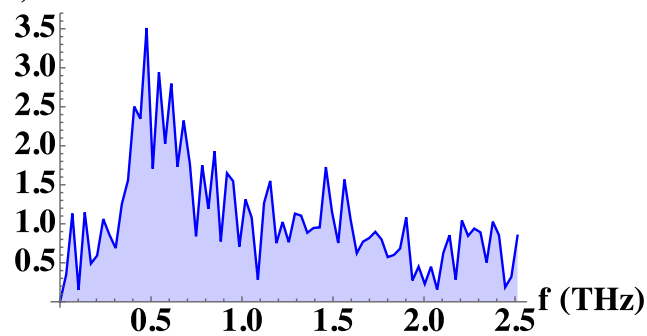

(f)

FIG. 20. Fourier spectra of measured structures in the $1.7 \mathrm{MeV}$ electron beam time: (a)-(e)—using 100-kV linac voltage and (f) using $200-\mathrm{kV}$ linac voltage. The vertical scale is in arbitrary units. Since phases of the harmonics are random, we plot only the amplitudes of the Fourier transform.

bunch with sufficient signal to noise level while maintaining a time resolution sufficient to resolve individual psecsize structures-because of the random nature of the density perturbation and broadband of the PCI using multiple bunches per snapshot or averaging multiple images were not options since it simply resulted in a smooth density distribution representing the envelope of the electron beam; (ii) obtaining proper focusing of a space-charge dominated electron beam in the horizontal plane for the best possible energy resolution [Eq. (B11)]. To a lesser degree, focusing was also important in the vertical plane, because the signal to noise ratio of the captured images depends on the vertical beam size.

With the LEBT lattice fixed to drive PCI saturation, we used three quadrupoles upstream of the 45-degree dipole to both optimize the time resolution and minimize the vertical beam size at the beam profile monitor to a level sufficient for accurate recording of the density modulation of saturated PCI [see Fig. 16(a)]. We estimated that with a voltage of $100 \mathrm{kV}$ we obtained a time resolution of $\sim 0.5 \mathrm{psec}$.
The captured images were analyzed off-line. Profiles were digitized using the free ImageJ software [133] and a Fourier analysis was performed using Mathematica [116]. Random density modulation structures resulted in random Fourier spectra.

As can be seen in the spectra of the six measured density profiles, shown in Fig. 20, structures do not repeat themselves from shot to shot and the Fourier spectra peaks jump from 0.35 to $0.55 \mathrm{THz}$. Hence, use of an average power spectrum, as in Fig. 16(b), is the only proper way of comparing our simulations with the experiment.

Figure 20(f) shows the spectrum of the density profile measured with $200-\mathrm{kV}$ linac voltage. It has a significantly higher level of noise, when compared with those measured using a $100-\mathrm{kV}$ voltage. The excessive noise, the limited contrast and reduced signal-to-noise ratio with the linac operating at $200 \mathrm{kV}$ and above were the reasons why most of our measurements were performed using the $100-\mathrm{kV}$ voltage. 


\section{APPENDIX C: THE CEC SRF GUN DRIVE LASER}

The drive laser for the CeC SRF gun (see Fig. 21(a)) comprises five main five main components: (i) low noise $10 \mu \mathrm{W}, 1064 \mathrm{~nm}$ iXBlue seed laser with an $8 \mathrm{Gs} / \mathrm{sec} 12-$ bit arbitrary pulse shaper [134], 78-kHz rep-rate; (ii) low noise, high $(60 \mathrm{~dB})$ gain $\mathrm{Nd}$ :YAG regenerative amplifier with an average output power of $10 \mathrm{~W}$; (iii) second harmonic (532 nm, green light) converter; (iv) Pockels cell and beam shutters for laser pulse train controls: trains have 1 to $10-\mathrm{Hz}$ rep-rates, 1 pulse to $\mathrm{cw}$ (full number of pulses) per train; (v) 40-meter long in-vacuum laser transport system to CeC SRF gun.

Since the laser has a relatively broadband optical spectrum, it can produce laser pulses with high frequency modulations. Therefore, this has to be eliminated as a possible source of the density modulation observed in our experiments. We performed careful studies of the laser settings to ensure that this was not the case.

The longitudinal intensity profile of the laser pulses can be modulated when the peak gain of the amplifier spectrum is located between two longitudinal modes of the seed laser, which are separated by about $50 \mathrm{GHz}$. If one of the longitudinal modes in the seed laser is lined up with the maximum gain of the amplifier, it receives most of the energy during amplification, thereby drowning out the other modes. If the gain peak is centered between two modes both receive about equal amounts of energy conserving the mode beating present in the seed pulse.

The output spectrum of the seed laser diode can be controlled by changing the diode temperature. When it was improperly tuned, we indeed observed a strong modulation

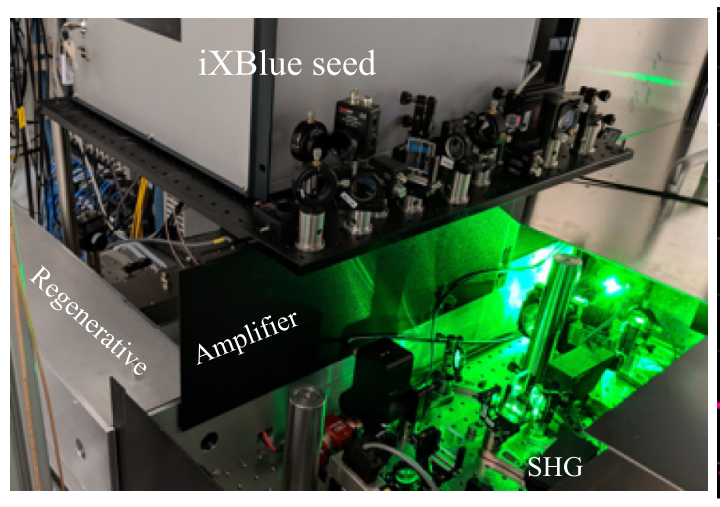

(a)

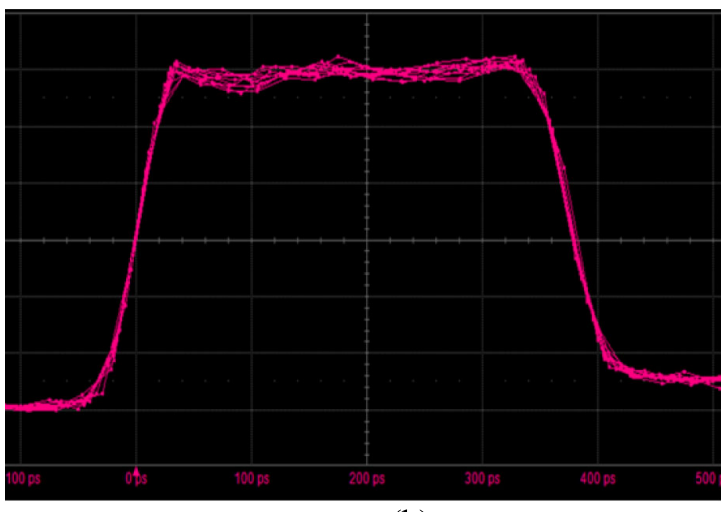

(b)

FIG. 21. (a) Photograph of the CeC SRF gun laser located in a dedicated temperature-controlled trailer outside of the RHIC tunnel. (b) Typical oscillogram of the green laser pulses with a typical FWHM duration of 375 psec used for these experiments.

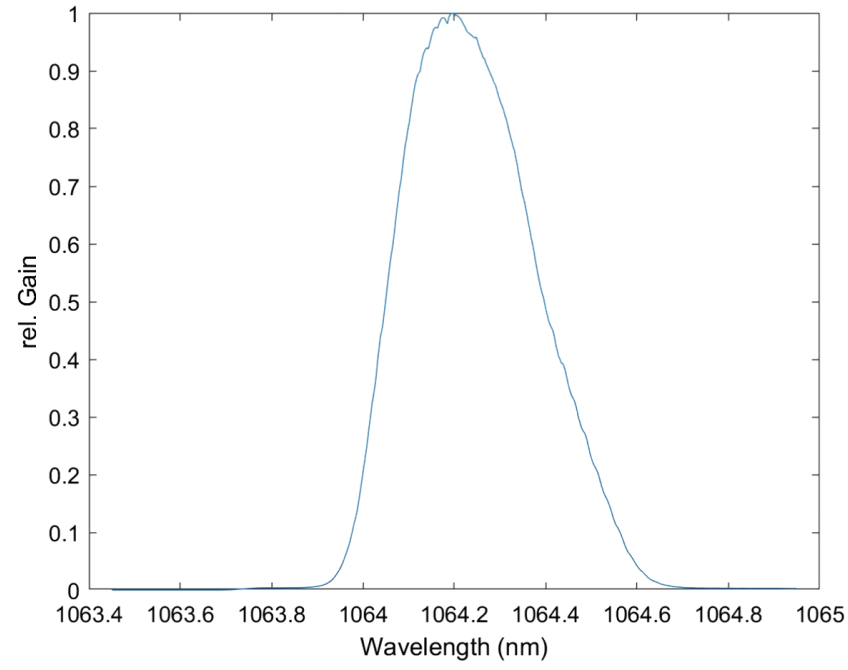

(a)

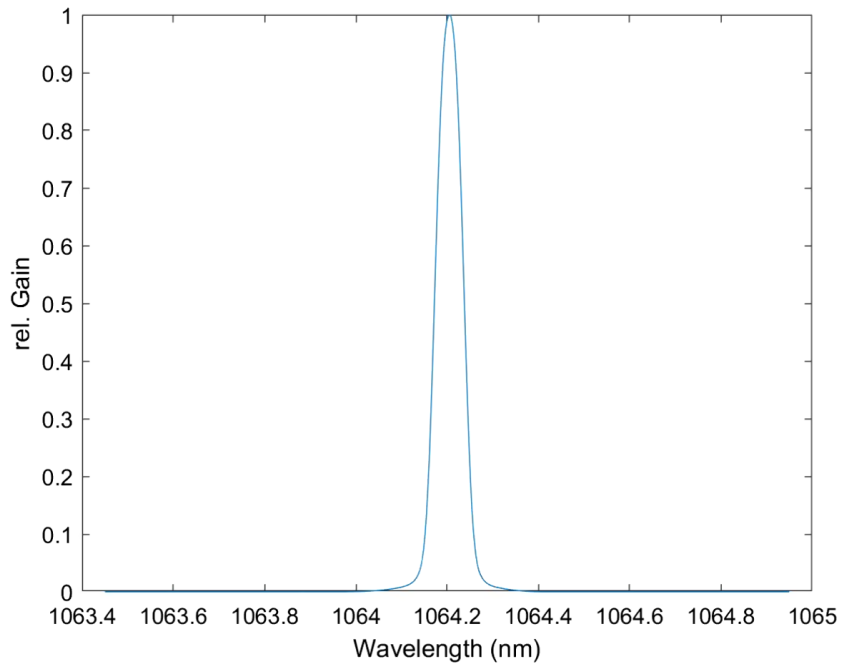

(b)

FIG. 22. (a) Normalized small-signal gain of the regenerative amplifier. (b) Measured spectrum of the drive laser before the second harmonic generation system. 


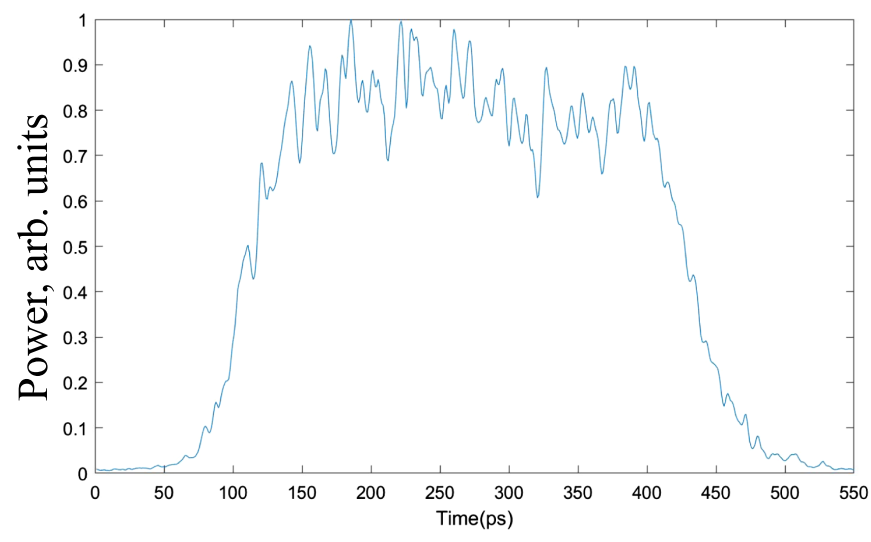

(a)

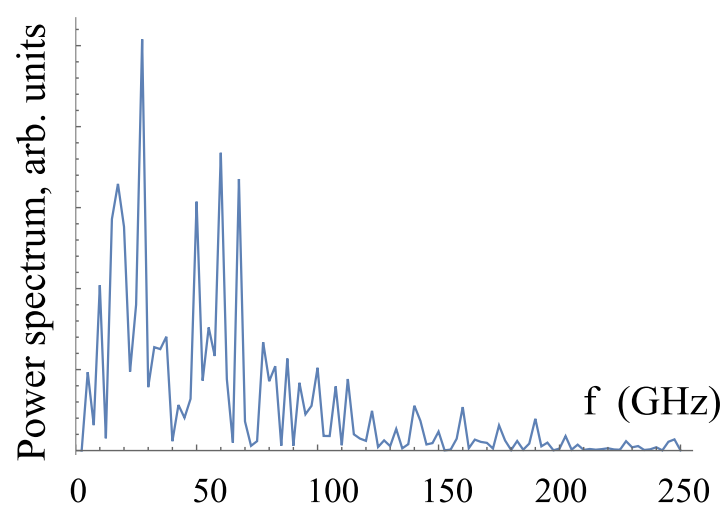

(b)

FIG. 23. (a) The profile of the second harmonic (green) laser pulse measured by a streak camera having a resolution of a few psec and (b) the spectrum of this pulse.

on the longitudinal laser pulse profile due to mode beating and a corresponding electron density modulation at frequencies of $\sim 50 \mathrm{GHz}$. When the seed laser was properly tuned, a single longitudinal mode dominated the amplified laser spectrum, which resulted in a reduced noise level below $100 \mathrm{GHz}$ (see Fig. 22).

For our experiment, we verified that the laser was properly tuned, and a single mode dominated the seed laser spectrum resulting in the green laser pulses with a noise level below $100 \mathrm{GHz}$ (see Fig. 23). These measurements assured us that the density modulation at frequencies of $\sim 0.5 \mathrm{THz}$ originated from the instability in the electron beam and not from the laser.

\section{APPENDIX D: EXAMINING OTHER POSSIBLE EXPLANATIONS}

In this Appendix we prove that there is no alternative to the plasma cascade instability, which can explain experimental observation of nearly saturated microbunching, shown in Figs. 15 and 16. Since we excluded wakefields and laser pulse structure as possible sources for the observe sub-THz density modulation, we examine two other suspects: energy spread induced by coherent synchrotron radiation (CSR) in the bending magnet and conventional microbunching instability (MBI) [79-97].

The CSR instability can be excluded because very low beam energy results in $0.007 \mathrm{THz}$ critical frequency of the synchrotron radiation spectrum from the 45-degree bending magnet. It means that at $0.5 \mathrm{THz}$ this radiation is suppressed astronomical 31 orders of magnitude and that CSR does not affect the beam. While it is a much weaker argument, we also confirmed this with CSR simulations using the code ELEGANT [135].

Similarly, we can eliminate the possibility that the observed data can be explained by conventional MBI [79-97]. According to the concept of conventional MBI, beam propagating in a straight section of linear accelerator could develop only energy modulation, and this energy modulation can be transferred into density modulation only when the beam trajectory is bent.

In our system such bending occurs only in the dipole magnet, which is located downstream of the linac introducing the time-dependent chirp. Because of the causality, illustrated by equation (B9), this time-dependent density modulation would not affect transverse beam profiles in our experiment. Hence, conventional MBI can affect observed profiles only through the time-dependent energy modulation.

It is well known that acceleration of electron beam freezes longitudinal motions and corresponding longitudinal temperature plummets to astronomically low levels [111]:

$$
k_{B} T_{\|}=\frac{1}{\beta_{o}^{2} \gamma_{o}^{3}} \frac{\left(k_{B} T_{\| \mathrm{cath}}\right)^{2}}{m c^{2}},
$$

where $k_{B} T_{\| \text {cath }}<0.25 \mathrm{eV}$ is the longitudinal temperature of electron beam at the gun's photocathode. At the exit of our 1.25 MV SRF gun, used for this experiment, the longitudinal temperature drops to $10^{-9} \mathrm{eV}$ level, corresponding to a minuscular relative rms energy spread $\sim 10^{-7}$. Other sources, which do not have THz frequency content, keep the relative rms slice energy spread at $10^{-4}$ level.

Hence, the high-frequency time-dependent energy modulation can only develop from the shot noise in electron beam current via plasma oscillation. It easy to show (see e.g., [136] and references therein) that measuring system with response time $\tau=1 / \sigma_{\omega}$ will observe longitudinal density modulation with relative rms amplitude of

$$
\sigma_{S N}=\sqrt{\frac{e}{2 \sqrt{\pi} I_{e} \cdot \tau}}
$$

This expression is derived for the cold electron beam and measurement system with the Gaussian kernel, with 
Gaussian frequency acceptance $\phi(\omega)=\exp \left(-\omega^{2} / 2 \sigma_{\omega}^{2}\right)$. In our case, with rms time resolution of 0.5 psec and peak current of 2.5 A relative density modulation from the Poisson shot noise would result in relative density modulation less than $2 \times 10^{-4}$-at least 3 orders of magnitude below the density modulation observed in our experiment. Naturally, in real beam this fluctuation will be reduced by the Landau damping.

Plasma oscillations would transfer the density modulation (D2) into energy modulation and vice versa. One can easily show, using Eqs. (2) and (B14), that resulting modulation with relative amplitude of

$$
\frac{\Delta \rho}{\rho}= \pm\left(\gamma_{o} \beta_{o}\right)^{2} \frac{m c^{2}}{\mathrm{eV}_{R F}} \frac{\omega_{p}}{\omega_{r f}} \sigma_{S N},
$$

where $\omega_{p}=c \sqrt{4 \pi n_{o} r_{c}}$ is the plasma frequency in the comoving frame (4). With beam parameters used in our experiment, the resulting relative modulation in the energy profile would be at $3 \times 10^{-3}$ level-again, well below modulation observed in the experiment. Again, this estimation is done for cold plasma, and in real beam this value will be reduced by the Landau damping term.

Hence, we conclude that conventional MBI concept fails to explain strong modulation of the energy spectrum, and the related transverse beam profiles on the YAG screen, observed in our experiment.

In contrast with the conventional MBI theory, the PCI concept does not assume that electron beam propagating in the straight section is stable. In this Appendix we showed that measured density modulation observed using PCI lattice exceeds prediction of conventional MBI theory by 2-3 orders of magnitude. Thus, we conclude that there is no an alternative explanation to our experimental demonstration of the newly discovered plasma-cascade instability.

[1] Challenges and Goals for Accelerators in the XXIst Century, edited by S. Myers and O. Bruning (World Scientific, Singapore, 2016).

[2] O. Brüning, P. Collier, P. Lebrun, S. Myers, R. Ostojic et al., LHC design report, CERN, Geneva, 2004, https:// cds.cern.ch/record/782076? ln=en.

[3] J. L. Abelleira Fernandez, C. Adolphsen, A. N. Akay, H. Aksakal, J. L. Albacete et al., A large hadron electron collider at CERN, J. Phys. G 39, 75001 (2012).

[4] ATLAS Collaboration, Observation of $H \rightarrow$ bb decays and $\mathrm{VH}$ production with the ATLAS detector, arXiv: 1808.08238.

[5] CMS Collaboration, Observation of Higgs Boson Decay to Bottom Quarks, Phys. Rev. Lett. 121, 121801 (2018).

[6] P. Emma et al., First lasing and operation of an ångstromwavelength free-electron laser, Nat. Photonics 4, 641 (2010).

[7] C. Pellegrini, X-ray free-electron lasers: From dreams to reality, Phys. Scr. T169, 014004 (2016).
[8] D. Pile, First light from SACLA, Nat. Photonics 5, 456 (2011).

[9] T. Ishikawa et al., A compact x-ray free-electron laser emitting in the sub-angstrom region, Nat. Photonics 6, 540 (2012).

[10] European XFEL, https://www.xfel.eu.

[11] H. Weise and W. Decking, Commissioning and first lasing of the European XFEL, 38th International FreeElectron Laser Conference, FEL 2017, Santa Fe, 2017 (JACoW Publishing, Geneva, Switzerland, 2017), p. 5, http://accelconf.web.cern.ch/fel2017/papers/moc03.pdf.

[12] In Soo Ko et al., Construction and commissioning of PAL-XFEL facility, Appl. Sci. 7, 479 (2017).

[13] C. J. Milne et al., Swiss FEL: The Swiss X-ray Free Electron Laser, Appl. Sci. 7, 720 (2017).

[14] A. Doerr, The new XFELs, Nat. Methods 15, 33 (2018)

[15] Pohang Accelerator Laboratory, http://pal.postech.ac.kr/ paleng/.

[16] P. Ball, Europe's X-ray laser fires up, Nature (London) 548, 507 (2017).

[17] Z. Huang and I. Lindau, SACLA hard-X-ray compact FEL, Nat. Photonics 6, 505 (2012).

[18] H.-S. Kang et al., Hard X-ray free-electron laser with femtosecond-scale timing jitter, Nat. Photonics 11, 708 (2017).

[19] C.-C. Kao, Challenges and opportunities for the next decade of XFELs, Nat. Rev. Phys. 2, 340 (2020).

[20] B. Eliasson and Chuan-Sheng Liu, An electromagnetic gamma-ray free electron laser, J. Plasma Phys. 79, 995 (2013).

[21] A. Accardi, J. L. Albacete, M. Anselmino et al., Electronion collider: The next QCD frontier, Eur. Phys. J. A 52, 268 (2016).

[22] J. L. A. Fernandez, C. Adolphsen, A. N. Akay, H. Aksakal, J. L. Albacete et al., A large hadron electron collider at CERN report on the physics and design concepts for machine and detector, J. Phys. G 39, 075001 (2012)

[23] D. Boer, M Diehl, R. Milner, R. Venugopalan, W. Vogelsang, A. Accardi et al., Gluons and the quark sea at high energies: Distributions, polarization, tomography, arXiv:1108.1713.

[24] K. J. Kim, Y. V. Shvyd'ko, and S. Reiche, A Proposal for an X-Ray Free-Electron Laser Oscillator with an EnergyRecovery Linac, Phys. Rev. Lett. 100, 244802 (2008).

[25] K. J. Kim and Y. V. Shvyd'ko, Tunable optical cavity for an X-ray free-electron-laser oscillator, Phys. Rev. ST Accel. Beams 12, 030703 (2009).

[26] V. N. Litvinenko, Optics-free FEL oscillator, in Proceedings of the FEL'2002 Conference, Argonne, IL, 2003 (Elsevier Science New York, 2003), p. II-23.

[27] V. N. Litvinenko, Y. Hao, D. Kayran, and D. Trbojevic, Optics-free x-ray FEL oscillator, in Proceedings of the 24th Particle Accelerator Conference, PAC-2011, New York, 2011 (IEEE, New York, 2011), p. 802.

[28] J. Rosenzweig, N. Barov, A. Murokh, E. Colby, and P. Colestock, Towards a plasma wake-field accelerationbased linear collider, Nucl. Instrum. Methods Phys. Res., Sect. A 410, 532 (1998). 
[29] C. B. Schroeder, E. Esarey, C. G. R. Geddes, C. Benedetti, and W. P. Leemans, Physics considerations for laserplasma linear colliders, Phys. Rev. ST Accel. Beams 13, 101301 (2010).

[30] J. Rosenzweig, Compact FEL based on Dielectric Wakefields, at the Towards a 5th Generation Light Sources Workshop (Avalon, Catalina Island, 2010).

[31] C. Jing, Wakefield in structures, AIP Conf. Proc. 1777, 020003 (2016).

[32] K. Nakajima, M. Kando, T. Kawakubo, T. Nakanishi, and A. Ogata, A table-top X-ray FEL based on the laser wakefield accelerator-undulator system, Nucl. Instrum. Methods Phys. Res., Sect. A 375, 593 (1996).

[33] I. Blumenfeld et al., Energy doubling of $42 \mathrm{GeV}$ electrons in a metre-scale plasma wakefield accelerator, Nature (London) 445, 741 (2007).

[34] S. Corde, C. Thaury, A. Lifschitz, G. Lambert, K. Ta Phuoc, X. Davoine, R. Lehe, D. Douillet, A. Rousse, and V. Malka, Observation of longitudinal and transverse selfinjections in laser-plasma accelerators, Nat. Commun. 4, 1501 (2013).

[35] N. Jain, T. M. Antonsen, and J. P. Palastro, Positron Acceleration by Plasma Wakefields Driven by a Hollow Electron Beam, Phys. Rev. Lett. 115, 195001 (2015).

[36] A. Zholents et al., A preliminary design of the collinear dielectric wakefield accelerator, Nucl. Instrum. Methods Phys. Res., Sect. A 829, 190 (2016).

[37] L. J. Laslett, On intensity limitations imposed by transverse space-charge effects in circular particle accelerators, BNL Report No. 7535, 1963, p. 154, http://lss.fnal.gov/ conf/C630610/p324.pdf.

[38] C. E. Nielsen and A. M. Sessler, Longitudinal space charge effects in particle accelerators, Rev. Sci. Instrum. 30, 80 (1959).

[39] S. Machida, Space-charge effects in low-energy proton synchrotrons, Nucl. Instrum. Methods Phys. Res., Sect. A 309, 43 (1991).

[40] S. G. Anderson, J. B. Rosenzweig, G. P. LeSage, and J. K. Crane, Space-charge effects in high brightness electron beam emittance measurements, Phys. Rev. ST Accel. Beams 5, 014201 (2002).

[41] A. V. Fedotov, Resonances and beam loss in high intensity rings, in Proceedings of the 2003 Particle Accelerator Conference, Portland, OR (IEEE, New York, 2003), p. 383.

[42] B. W. Montague, Forth-order coupling resonance excited by space-charge forces in synchrotron, CERN Report No. 68-38, 1968.

[43] I. Hofmann, Stability of anisotropic beams with space charge, Phys. Rev. E 57, 4713 (1998).

[44] V. N. Litvinenko and G. Wang, Compensating tune spread induced by space charge in bunched beams, Phys. Rev. ST Accel. Beams 17, 114401 (2014).

[45] S. Machida, Space-charge-induced resonances in a synchrotron, Nucl. Instrum. Methods Phys. Res., Sect. A 384, 316 (1997).

[46] I. Hofmann and K. Beckert, Resonance crossing in the presence of space charge, in Proceedings of the 11th Particle Accelerator Conference, PAC-1985, Vancouver, BC, Canada, 1985 (IEEE, New York, 1985), p. 2264.
[47] F. Sacherer, Transverse space-charge effects in circular accelerators, Ph.D. thesis, University of California, Berkeley, 1968 [LNBL Report No. UCRL-18454].

[48] R. L. Gluckstern, Oscillation modes in two dimensional beams, in Proceedings of the 1970 Proton Linear Accelerator Conference, Batavia, IL, 1970, p. 811, https:// inspirehep.net/files/e26aec56ff62e4f2ea9429be2022936c

[49] I. Hofmann et al., Review of beam dynamics and space charge resonances in high intensity linacs, in Proceedings of the 8th European Particle Accelerator Conference, Paris, 2002 (EPS-IGA and CERN, Geneva, 2002), p. 74.

[50] A. V. Fedotov, J. Holmes, and R. L. Gluckstern, Instabilities of high-order beam modes driven by space-charge coupling resonances, Phys. Rev. ST Accel. Beams 4, 084202 (2001).

[51] J. S. O'Connell, T. P. Wangler, R. S. Mills, and K. R. Crandall, Beam halo formation from space-charge dominated beams in uniform focusing channel, in Proceedings of the Particle Accelerator Conference, Washington, DC, 1993 (IEEE, Piscataway, NJ, 1993), p. 3657.

[52] J. Qiang and R. D. Ryne, Beam halo studies using a threedimensional particle-core model, Phys. Rev. ST Accel. Beams 3, 064201 (2000).

[53] J. M. Lagniel, On halo formation from space-charge dominated beams, Nucl. Instrum. Methods Phys. Res., Sect. A 345, 46 (1994); 345, 405 (1994).

[54] A. Riabko, M. Ellison, X. Kang, S. Lee, D. Li, J. Liu, X. Pei, and L. Wang, Hamiltonian formalism for space charge dominated beams in a uniform focusing channel, Phys. Rev. E 51, 3529 (1995).

[55] M. S. Livingston, Design Study for a 15-GeV Accelerator, MIT Report No. 60, 1953, p. 154.

[56] H. Okamoto and K. Yokoya, Parametric resonances in intense one-dimensional beams propagating through a periodic focusing channel, Nucl. Instrum. Methods Phys. Res., Sect. A 482, 51 (2002).

[57] I. Hofmann, L. J. Laslett, L. Smith, and I. Haber, Stability of the Kapchinskij-Vladimirskij (K-V) distribution in long periodic transport systems, Part. Accel. 13, 145 (1983).

[58] J. Struckmeier and M. Reiser, Theoretical studies of envelope oscillations and instabilities of mismatched intense charged-particle beams in periodic focusing channels, Part. Accel. 14, 227 (1984).

[59] Handbook of Accelerator Physics, and Engineering, edited by A. Chao and M. Tigner, 2nd ed. (World Scientific, Singapore, 2002), p. 128.

[60] J. B. Murphy, An introduction to coherent synchrotron radiation in storage rings, ICFA Beam Dynam. Newsletter 35, 20 (2004), https://icfa-usa.jlab.org/archive/newsletter/ icfa_bd_nl_35.pdf.

[61] Ya. S. Derbenev, J. Rossbach, E. L. Saldin, and V. D. Shiltsev, Microbunch radiative tail-head interaction, Report No. TESLA FEL-Report 1995-05, DESY, Hamburg, Germany, 1995, https://flash.desy.de/sites2009/site_vuvfel/ content/e403/e1642/e1488/e1490/infoboxContent2098/ tesla-fel1995-05.pdf.

[62] J. B. Murphy, S. Krinsky, and R. L. Gluckstern, Longitudinal wakefield for synchrotron radiation, in Proceedings of the Particle Accelerator Conference, Dallas, TX, 1995 (IEEE, New York, 1995), Vol. 5, pp. 2980-2982. 
[63] E. L. Saldin, E. A. Schneidmiller, and M. V. Yurkov, On the coherent radiation of an electron bunch moving in an arc of a circle, Nucl. Instrum. Methods Phys. Res., Sect. A 398, 373 (1997).

[64] C. Mayers and G. Hoffstaetter, Exact 1D model for coherent synchrotron radiation with shielding and bunch compression, Phys. Rev. ST Accel. Beams 12, 024401 (2009).

[65] V. Yakimenko, M. Fedurin, V. Litvinenko, A. Fedotov, D. Kayran, and P. Muggli, Experimental Observation of Suppression of Coherent-Synchrotron-RadiationInduced Beam-Energy Spread with Shielding Plates, Phys. Rev. Lett. 109, 164802 (2012).

[66] S. Antipov, C. Jing, M. Fedurin, W. Gai, A. Kanareykin, K. Kusche, P. Schoessow, V. Yakimenko, and A. Zholents, Experimental Observation of Energy Modulation in Electron Beams Passing through Terahertz Dielectric Wakefield Structures, Phys. Rev. Lett. 108, 144801 (2012).

[67] G. P. Williams, Filling the THz gap-High power sources and applications, Rep. Prog. Phys. 69, 301 (2006).

[68] C. Sung, S. Ya. Tochitsky, S. Reiche, J. B. Rosenzweig, C. Pellegrini, and C. Joshi, Seeded free-electron and inverse free-electron laser techniques for radiation amplification and electron microbunching in the terahertz range, Phys. Rev. ST Accel. Beams 9, 120703 (2006).

[69] D. Ratner, R. Abela, J. Amann, C. Behrens, D. Bohler et al., Experimental Demonstration of a Soft X-Ray SelfSeeded Free-Electron Laser, Phys. Rev. Lett. 114, 054801 (2015).

[70] E. Allaria, R. Appio, L. Badano, W. A. Barletta, S. Bassanese et al., Highly coherent and stable pulses from the FERMI seeded free-electron laser in the extreme ultraviolet, Nat. Photonics 6, 699 (2012).

[71] V. N. Litvinenko and Y. S. Derbenev, Coherent Electron Cooling, Phys. Rev. Lett. 102, 114801 (2009).

[72] D. Ratner, Microbunched Electron Cooling for HighEnergy Hadron Beams, Phys. Rev. Lett. 111, 084802 (2013).

[73] V. N. Litvinenko and Y.S. Derbenev, FELs and highenergy electron cooling, in Proceedings of the 29th Free Electron Laser Conference, Novosibirsk, Russia (BINP, Novosibirsk, 2007), p. 268.

[74] V. N. Litvinenko, Advances in coherent electron cooling, in Proceedings of COOL 2013 Workshop, 2013, Mürren, Switzerland (JACoW Publishing, 2013), p. 175, http:// accelconf.web.cern.ch/COOL2013/papers/tham2ha02 .pdf.

[75] V. N. Litvinenko, G. Wang, D. Kayran, Y. Jing, J. Ma, and I. Pinayev, Plasma-cascade microbunching amplifier and coherent electron cooling of a hadron beams, arXiv:1802.08677, https://arxiv.org/pdf/1802.08677.pdf.

[76] A. Chao, E. Granados, X. Huang, D. Ratner, and H.-W. Luo, High power radiation sources using the steady-state microbunching mechanism, in Proceedings of IPAC2016 Busan, Korea (JACoW Publishing, 2016), p. 1048, http:// accelconf.web.cern.ch/ipac2016/papers/tuoca01.pdf.

[77] D. F. Ratner and A. W. Chao, Steady-State Microbunching in a Storage Ring for Generating Coherent Radiation, Phys. Rev. Lett. 105, 154801 (2010).
[78] Y. Jiao, D. F. Ratner, and A. W. Chao, Terahertz coherent radiation from steady-state microbunching in storage rings with X-band radio-frequency system, Phys. Rev. ST Accel. Beams 14, 110702 (2011).

[79] R. A. Lacey, Indications for a Critical End Point in the Phase Diagram for Hot and Dense Nuclear Matter, Phys. Rev. Lett. 114, 142301 (2015).

[80] E. L. Saldin, E. A. Schneidmiller, and M. V. Yurkov, Klystron instability of a relativistic electron beam in a bunch compressor, Nucl. Instrum. Methods Phys. Res., Sect. A 490, 1 (2002).

[81] S. Heifets, S. Krinsky, and G. Stupakov, Coherent synchrotron radiation instability in a bunch compressor, Phys. Rev. ST Accel. Beams 5, 064401 (2002).

[82] E. A. Schneidmiller and M. V. Yurkov, Using the longitudinal space charge instability for generation of vacuum ultraviolet and x-ray radiation, Phys. Rev. ST Accel. Beams 13, 110701 (2010).

[83] T. Shaftan and Z. Huang, Experimental characterization of a space charge induced modulation in high-brightness electron beam, Phys. Rev. ST Accel. Beams 7, 080702 (2004)

[84] M. Venturini, Microbunching instability in single-pass systems using a direct two-dimensional Vlasov solver, Phys. Rev. ST Accel. Beams 10, 104401 (2007).

[85] M. Venturini, Models of longitudinal space-charge impedance for microbunching instability, Phys. Rev. ST Accel. Beams 11, 034401 (2008).

[86] D. Ratner, Z. Huang, and A. Chao, Three-dimensional analysis of longitudinal space charge microbunching starting from shot noise, in Proceedings of 30th International Free Electron Laser Conference (FEL 2008), 24-29 August 2008, Gyeongju, Republic of Korea (JACoW Publishing, 2008), http://accelconf.web.cern.ch/ FEL2008/papers/tupph041.pdf.

[87] M. Dohlus, E. A. Schneidmiller, and M. V. Yurkov, Generation of attosecond soft $\mathrm{x}$-ray pulses in a longitudinal space charge amplifier, Phys. Rev. ST Accel. Beams 14, 090702 (2011).

[88] A. Gover and E. Dyunin, Collective-Interaction Control and Reduction of Optical Frequency Shot Noise in Charged-Particle Beams, Phys. Rev. Lett. 102, 154801 (2009).

[89] R. Akre, D. Dowell, P. Emma, J. Frisch, S. Gilevich et al., Commissioning the Linac Coherent Light Source injector, Phys. Rev. ST Accel. Beams 11, 030703 (2008).

[90] A. Marinelli and J. B. Rosenzweig, Microscopic kinetic analysis of space-charge induced optical microbunching in a relativistic electron beam, Phys. Rev. ST Accel. Beams 13, 110703 (2010).

[91] A. Marinelli, E. Hemsing, M. Dunning, D. Xiang, S. Weathersby, F. O'shea, I. Gadjev, C. Hast, and J. B. Rosenzweig, Generation of Coherent Broadband Photon Pulses with a Cascaded Longitudinal Space-Charge Amplifier, Phys. Rev. Lett. 110, 264802 (2013).

[92] Z. Huang and K.-J. Kim, Formulas for coherent synchrotron radiation microbunching in a bunch compressor chicane, Phys. Rev. ST Accel. Beams 5, 074401 (2002).

[93] Z. Huang, M. Borland, P. Emma, J. Wu, C. Limborg, G. Stupakov, and J. Welch, Suppression of microbunching 
instability in the linac coherent light source, Phys. Rev. ST Accel. Beams 7, 074401 (2004).

[94] D. Ratner. Much ado about microbunching: Coherent bunching in high brightness electron beams, Ph.D. thesis, Department of Applied Physics, Stanford University, 2011, https://stacks.stanford.edu/file/druid:ng211kg5080/ ThesisRoot-augmented.pdf.

[95] A. Marinelli, E. Hemsing, and J. B. Rosenzweig, Three dimensional analysis of longitudinal plasma oscillations in a thermal relativistic electron beam, Phys. Plasmas 18, 103105 (2011).

[96] V. N. Litvinenko and G. Wang, Relativistic effects in microbunching, in, Proceedings of FEL2014, Basel, Switzerland, August 25-29, 2014, p. 790, http://accelconf .web.cern.ch/FEL2014/papers/thp035.pdf.

[97] D. Ratner, Microbunching instability: Review of current models, observations, and solutions, ICFA Beam Dynamics Newsletter 59, 112 (2012), https://icfa-usa.jlab.org/ archive/newsletter/icfa_bd_nl_59.pdf.

[98] A. Fedotov, I. Hofmann, R. L. Gluckstern, and H. Okamoto, Parametric collective resonances and spacecharge limit in high-intensity rings, Phys. Rev. ST Accel. Beams 6, 094201 (2003).

[99] V. N. Litvinenko and G. Wang, Theory of plasma-cascade instability, arXiv:2101.04103.

[100] D. R. Nicholson, Introduction in Plasma Theory (John Wiley \& Sons, New York, 1983).

[101] R. C. Davidson, Physics of Nonneutral Plasmas (Addison- Wesley, Reading, MA, 1990).

[102] This method was introduced to us by Y.S. Derbenev (JLab) (private communication).

[103] E. D. Courant and H. S. Snyder, Theory of the alternatinggradient synchrotron, Ann. Phys. (Paris) 3, 1 (1958).

[104] A. A. Kolomenskyand A. N. Lebedev, Theory of Cyclic Accelerators (North-Holland, Amsterdam, 1966).

[105] S. Y. Lee, Accelerator Physics (World Scientific, Singapore, 2011).

[106] H. Wiedemann, Particle Accelerator Physics (Springer, New York, 2007).

[107] L. D. Landau and E. M. Lifshitz, Classical Mechanics (Elsevier Science, New York, 1976).

[108] L. Landau, On the vibration of the electronic plasma, J. Phys. (USSR) 10, 25 (1946) [J. Exp. Theor. Phys. 16, 574 (1946)]; Reproduced in Collected papers of L. D. Landau, edited and with an introduction by D. ter Haar (Pergamon Press, New York, 1965), pp. 445-460; and in L. D. Landau, Men of Physics Vol. 2, edited by D. ter Haar (Pergamon Press, New York, 1965).

[109] Collected Papers of L. D. Landau, edited and with an introduction by D. ter Haar (Pergamon Press, New York, 1965), p. 445.

[110] I. M. Kapchinsky and V. V. Vladimirsky, Limitations of proton beam current in a strong focusing linear accelerator associated with the beam space charge, in Proceedings of the International Conference on High Energy Accelerators and Instrumentation (CERN Scientific Information Service, Geneva, 1959), p. 274.

[111] M. Reiser, Theory and Design of Charged Particle Beams (John Wiley \& Sons, New York, 2008).
[112] G. Wang, Coherent electron cooling and two stream instabilities due to electron cooling, Ph.D. thesis, State University of New York at Stony Brook, 2008.

[113] A. A. Vlasov, Vibrational properties of the electronic gas, Zh. Eksp. Teor. Fiz. 8, 291 (1938), https://iopscience.iop .org/article/10.1070/PU1968v010n06ABEH003709/ meta.

[114] G. Wang and M. Blaskiewicz, Dynamics of ion shielding in an anisotropic electron plasma, Phys. Rev. E 78, 026413 (2008).

[115] G. Wang, M. Blaskiewicz, and V. N. Litvinenko, Simulation of ion beam under coherent electron cooling, in Proceedings of IPAC 2016, Busan, Korea, 2016, p. 1247, http://accelconf.web.cern.ch/AccelConf/ipac2016/ papers/tupmr009.pdf.

[116] Wolfram Research, Inc., Mathematica, Version 11.3, Champaign, IL (2018).

[117] E. Forest, Fourth-order symplectic integrator, Reports No. SLAG-PUB-5071 and No. LBL-27662, 1989.

[118] G. Wang, V. N. Litvinenko, and J. Ma, Predicting the performances of coherent electron cooling with plasma cascade amplifier, in Proceedings of the 10th International Particle Accelerator Conference, IPAC2019, Melbourne, Australia, p. 2150, http://accelconf.web.cern.ch/ipac2019/ papers/tupts099.pdf.

[119] V. N. Litvinenko, Analytical tools in accelerator physics, C-A/AP/406 note, 2010, https://www.bnl.gov/ isd/documents/74289.pdf.

[120] A. J. Dragt, Lie methods for nonlinear dynamics with applications to accelerator physics, University of Maryland, Center for Theoretical Physics, Department of Physics, 1997, https://www.physics.umd.edu/dsat/docs/ Book19Nov2020.pdf.

[121] X. Wang, R. Samulyak, J. Jiao, and K. Yu, Adaptive particle-in-cloud method for optimal solutions to VlasovPoisson equation, J. Comput. Phys. 316, 682 (2016).

[122] K. Yu and R. Samulyak, SPACE code for beam-plasma interaction, in Proceedings the sixth International Particle Accelerator Conference, IPAC'15, Richmond, Virginia, USA, May 3-8 (JACoW Publishing, 2015), p. 728, MOPMN012, http://accelconf.web.cern.ch/ IPAC2015/papers/mopmn012.pdf.

[123] Jun Ma, Gang Wang, and Vladimir Litvinenko, Simulations of coherent electron cooling with two types of amplifiers, Int. J. Mod. Phys. 34, 1942029 (2019).

[124] Vladimir N. Litvinenko, Zeynep Altinbas, Jean Clifford Brutus, Anthony Di Lieto, David Gassner et al., Coherent electron cooling experiment at RHIC: Status and plans, in Proceedings of the 12th Workshop on Beam Cooling and Related Topics, COOL2019, Novosibirsk, Russia, pp. 35-40, http://accelconf.web.cern.ch/cool2019/papers/ tuz01.pdf.

[125] V. N. Litvinenko, Z. Altinbas, R. Anderson, S. Belomestnykh, C. Boulware et al., Commissioning of FEL-based coherent electron cooling system, in Proceedings of the 38th International Free Electron Laser Conference (FEL'17), Santa Fe, NM (JACoW Publishing, 2017), p. 132., http://accelconf.web.cern.ch/fel2017/papers/mop041 .pdf. 
[126] http://www.cst.com, CST-Computer Simulation Technology (2017).

[127] I. Zagorodnov, Wakefield Code ECHO, 2016, https:// indico.cern.ch/event/459623/contributions.

[128] Azimuthal Beam Cavity Interaction - ABCI code, https:// abci.kek.jp/abci.htm

[129] Ji Qiang, Impact-T: A 3D parallel particle tracking code in time domain, LBNL, https://amac.lbl.gov/ jiqiang/ IMPACT-T/index.html.

[130] NERSC, https://www.nersc.gov.
[131] J. J. Sylvester, On the equation to the secular inequalities in the planetary theory, Philos. Mag. Ser. 5 16, 267 (1883).

[132] R. A. Horn and C. R. Johnson, Topics in Matrix Analysis (Cambridge University Press, Cambridge, England, 1991).

[133] ImageJ, https://imagej.nih.gov/ij/index.html.

[134] iXblue, https://www.ixblue.com.

[135] M. Borland, Argonne National Laboratory Advanced Photon Source Report No. LS-287, 2000.

[136] A. Gover, A. Nause, E. Dyunin, and M. Fedurin, Beating the shot-noise limit, Nat. Phys. 8, 877 (2012). 